\section{Biofouling}

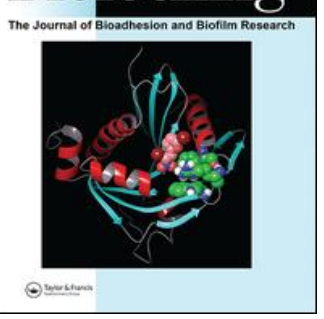

Biofouling

The Journal of Bioadhesion and Biofilm Research

\title{
Extracellular DNA and lipoteichoic acids interact with exopolysaccharides in the extracellular matrix of Streptococcus mutans biofilms
}

Midian C. Castillo Pedraza, Tatiana F. Novais, Roberta C. Faustoferri, Robert G. Quivey Jr., Anton Terekhov, Bruce R. Hamaker \& Marlise I. Klein

To cite this article: Midian C. Castillo Pedraza, Tatiana F. Novais, Roberta C. Faustoferri, Robert G. Quivey Jr., Anton Terekhov, Bruce R. Hamaker \& Marlise I. Klein (2017) Extracellular DNA and lipoteichoic acids interact with exopolysaccharides in the extracellular matrix of Streptococcus mutans biofilms, Biofouling, 33:9, 722-740, DOI: 10.1080/08927014.2017.1361412

To link to this article: https://doi.org/10.1080/08927014.2017.1361412

View supplementary material $₫$

Published online: 25 Sep 2017.

Submit your article to this journal $₫$

LIII Article views: 265

View Crossmark data [ᄌ

Citing articles: 8 View citing articles 지 


\title{
Extracellular DNA and lipoteichoic acids interact with exopolysaccharides in the extracellular matrix of Streptococcus mutans biofilms
}

\author{
Midian C. Castillo Pedraza a, Tatiana F. Novais ${ }^{a}$, Roberta C. Faustoferrib, Robert G. Quivey Jr. ${ }^{b}$ D, Anton Terekhov ${ }^{c}$, \\ Bruce R. Hamaker' and Marlise I. Klein ${ }^{\mathrm{a}}$ (D) \\ aDepartment of Dental Materials and Prosthodontics, School of Dentistry, São Paulo State University (Unesp), Araraquara, Brazil; ${ }^{b}$ Center for \\ Oral Biology, University of Rochester, Rochester, NY, USA; 'Whistler Center for Carbohydrate Research, Purdue University, West Lafayette, IN, USA
}

ABSTRACT

Streptococcus mutans-derived exopolysaccharides are virulence determinants in the matrix of biofilms that cause caries. Extracellular DNA (eDNA) and lipoteichoic acid (LTA) are found in cariogenic biofilms, but their functions are unclear. Therefore, strains of $S$. mutans carrying single deletions that would modulate matrix components were used: eDNA - $\Delta l y t S$ and $\Delta l y t T$; LTA - $\Delta d l t A$ and $\Delta d I t D ;$ and insoluble exopolysaccharide $-\Delta g t f B$. Single-species (parental strain S. mutans UA 159 or individual mutant strains) and mixed-species (UA159 or mutant strain, Actinomyces naeslundii and Streptococcus gordonii) biofilms were evaluated. Distinct amounts of matrix components were detected, depending on the inactivated gene. eDNA was found to be cooperative with exopolysaccharide in early phases, while LTA played a larger role in the later phases of biofilm development. The architecture of mutant strains biofilms was distinct (vs UA159), demonstrating that eDNA and LTA influence exopolysaccharide distribution and microcolony organization. Thus, eDNA and LTA may shape exopolysaccharide structure, affecting strategies for controlling pathogenic biofilms.
ARTICLE HISTORY

Received 26 January 2017 Accepted 25 July 2017

\section{KEYWORDS}

Biofilms; Streptococcus mutans; extracellular matrix; exopolysaccharides; eDNA; lipoteichoic acids

\section{Introduction}

Dental caries is a public health problem worldwide and a diet- and biofilm-dependent disease (Selwitz et al. 2007; Kassebaum et al. 2015; Bowen 2016). Biofilms are dynamic microbial communities enmeshed by a three-dimensional (3-D) extracellular matrix (ECM) (Branda et al. 2005; Flemming and Wingender 2010). Exopolysaccharides (EPS) are essential components in the assembly of the ECM of cariogenic biofilms (Paes Leme et al. 2006; Bowen and Koo 2011), and are also a virulence determinant (Koo et al. 2013). The ECM rich in EPS acts as a barrier that provides acidic niches where acidogenic and aciduric microorganisms thrive (Xiao et al. 2012), leading to the acid dissolution of enamel. In addition, extracellular DNA (eDNA) and lipoteichoic acids (LTA) are detected in high amounts in cariogenic biofilms in the presence of sucrose and starch (as reviewed by Klein et al. 2015). However, the roles of eDNA and LTA in the construction of the extracellular matrix are not yet well understood.

EPS are synthesized by glycosyltransferase enzymes (Gtfs) and fructosyltransferases (Ftfs) when dietary sucrose is present (Vacca-Smith et al. 1996; Bowen and Koo 2011). Furthermore, starch hydrolysates affect the glycosyl linkages of glucans and the 3-D distribution of glucans within biofilms (Klein et al. 2009). The Gtf exoenzymes produced by Streptococcus mutans synthesize water-insoluble and -soluble glucans; $S$. mutans produces at least three distinct enzymes (encoded by $g t f B, g t f C$ and gtfD genes; as reviewed by Bowen and Koo 2011) that coordinate the assembly of an EPS-rich ECM (Xiao et al. 2012).

eDNA and LTA could help in the construction of the ECM by increasing the synthesis of glucan (Kuramitsu et al. 1980; Ciardi et al. 1981; Chiu and Baker 1994), and promoting microbial adhesion to surfaces (Ciardi et al. 1977; Gross et al. 2001; Das et al. 2010). eDNA from $S$. mutans is a byproduct of autolysis and can also be actively secreted via microvesicles (Perry et al. 2009; Liao et al. 2014), while LTA could be released to the ECM during cell lysis and cell wall remodeling. Both eDNA and LTA biomolecules can confer a negative charge to the ECM, which could influence its diffusion properties (Melvaer et al. 1972, 1974; Melsen et al. 1979; Pratten et al. 1998), and thereby affect the activity of cationic antimicrobial or anti-biofilm agents (Xiao et al. 2012).

The presence of dietary sucrose and starch may increase the release of eDNA in the ECM, due to elevated

\section{CONTACT Marlise I. Klein mklein@foar.unesp.br}

(1) The supplemental material for this paper is available online at https://doi.org/10.1080/08927014.2017.1361412

C 2017 Informa UK Limited, trading as Taylor \& Francis Group 
expression of $l y t S T$ and $c c p A$ during biofilm formation by S. mutans (Klein et al. 2010). The two-component system LytST is required to activate the expression of $\operatorname{lrg} A B$, which are part of the $S$. mutans arsenal that control biofilm formation and autolysis (Ahn et al. 2010). The expression of lytST and $\operatorname{lrg} A B$ is regulated by the availability of carbohydrates via CcpA (catabolite control protein A) (Ahn et al. 2010); thus, the abundance of available dietary sucrose and starch could elicit this mechanism. The expression of $g t f B$ (which synthesizes water-insoluble EPS) was also induced in biofilms formed in the presence of sucrose and starch (Klein et al. 2009, 2010). In addition, eDNA interacting with GtfB influences EPS production, colonization by $S$. mutans and possibly the construction of the ECM (Klein et al. 2010, 2015; Liao et al. 2014).

The mechanism by which LTA is synthesized has been described for some species, but not for $S$. mutans (Reichmann and Gründling 2011; Denapaite et al. 2012). However, it is known that the dltABCD operon is required for the addition of $\mathrm{D}$-alanine residues (Neuhaus and Baddiley 2003) during the formation of LTA; these residues affect the adhesion processes and biofilm formation (Spatafora et al. 1999; Gross et al. 2001). Furthermore, S. mutans proteins encoded by the dltA and dltD genes are abundant while the ECM is being actively built during the early stages of cariogenic biofilm formation (Klein et al. 2012).

Understanding how eDNA, LTA and EPS matrix components interact and influence the construction of biofilms may help the development of targeted therapies to prevent and remove cariogenic biofilms. Therefore, the aim of this study was to investigate how eDNA and LTA, in conjunction with EPS, impact the structural and functional properties of the cariogenic biofilm matrix.

\section{Material and methods}

\section{Experimental design}

An in vitro study was conducted using single- and mixed-species biofilm models based on a batch culture approach with saliva-coated hydroxyapatite (HA) disks as the substratum. The mixed-species system was

Table 1. S. mutans strains used in the study.

\begin{tabular}{|c|c|c|c|}
\hline $\begin{array}{l}\text { Streptococcal } \\
\text { strains }\end{array}$ & $\begin{array}{l}\text { Locus tag of } \\
\text { targeted gene }\end{array}$ & $\begin{array}{l}\text { Putative mod- } \\
\text { ulation of ECM } \\
\text { components }\end{array}$ & References \\
\hline UA159 & - & All & Ajdić et al. 2002 \\
\hline$\Delta g t f B$ & SMU_1004 & Insoluble EPS & $\begin{array}{l}\text { Dr .Robert A. } \\
\text { Burne }\end{array}$ \\
\hline$\Delta / y t T$ & SMU_576 & eDNA & Chatfield et al. \\
\hline Dlyts & SMU_577 & & 2005 \\
\hline$\Delta d l t A$ & SMU_1691 & LTA & Quivey et al. 2015 \\
\hline$\Delta d l t D$ & SMU_1688 & & \\
\hline
\end{tabular}

designed to mimic the formation of biofilms according to the 'ecological plaque-biofilm' concept (Marsh 2003), using S. gordonii instead of Streptococcus oralis (as detailed by Koo et al. 2010; Xiao et al. 2012). The study was approved by the Institutional Ethical Committee (CAAE: 31717914.3.0000.5416), because human saliva was used to perform in vitro experiments.

The factors to be examined were matrix component modulation by $S$. mutans strains (parental strain and single gene deletion strains; Table 1), and biofilm developmental phase in two types of biofilm cultures (singleand mixed-species), generating 12 experimental groups in which to assay biomass, population, and biochemical characteristics: (1) S. mutans UA159 (parental strain) at $67 \mathrm{~h},(2) \Delta g t f B$ at $67 \mathrm{~h},(3) \Delta l y t S$ at $67 \mathrm{~h},(4) \Delta l y t T$ at $67 \mathrm{~h}$, (5) $\Delta d l t A$ at $67 \mathrm{~h},(6) \Delta d l t D$ at $67 \mathrm{~h},(7) S$. mutans UA159 at $115 \mathrm{~h},(8) \Delta g t f B$ at $115 \mathrm{~h},(9) \Delta l y t S$ at $115 \mathrm{~h},(10) \Delta l y t \mathrm{~T}$ at $115 \mathrm{~h},(11) \Delta d l t A$ at $115 \mathrm{~h}$, and (12) $\Delta$ dlt D at $115 \mathrm{~h}$ ( $n=6$ for single-species biofilms at 67 and $115 \mathrm{~h}$, and for mixed-species biofilms at $115 \mathrm{~h}$ from three independent cultures; $n=10$ for mixed-species biofilms at $67 \mathrm{~h}$ ). In addition, distinct time points were evaluated to determine the microbial population of mixed-species over time (at $29,43,53,67,77,91,101$, and $115 \mathrm{~h} ; n=6$ for all time points except for $67 \mathrm{~h}$, where $n=10$ ). Three or five independent experiments were carried out in duplicate and the data were statistically analyzed according to the factorial design of this study, considering each HA disk as a statistical block ( $n=6$ or $n=10)$. The disks were randomly assigned for each experimental group. Biochemical analyses of the ECM were performed to verify how inactivation of specific genes influenced biofilm development, and consequent pathogenic potential. The authors' hypothesis was that EDNA and LTA would cooperate with EPS in the extracellular matrix of $S$. mutans biofilms, increasing the structural and functional complexity of those biofilms.

Furthermore, glycosyl linkage analysis of the water-soluble and -insoluble exopolysaccharides in the ECM of mixed-species biofilms were performed using a pool of 12 biofilm samples formed by each $S$. mutans strain to verify whether the modulation of specific ECM components affected EPS biochemical structure. Moreover, during biofilm growth, the culture medium was changed twice daily and the $\mathrm{pH}$ values of spent medium were measured. Confocal laser scanning microscopy (CLSM) was used to visualize and quantify the structural components (EPS and bacteria) of the biofilms.

\section{Bacterial strains}

Streptococcus mutans UA159, serotype c(ATCC 700610), a proven cariogenic organism, was the parental strain used in this study, from which deletion strains were created. 
S. mutans UA159 (parental strain) and mutant strains carrying deletions in $l y t S$, $l y t T$, dlt $A$ and $d l t D$ were used to modulate the presence of eDNA or LTA in the matrix (Table 1). The deletion strains for $l y t S, l y t T$ (Chatfield et al. 2005), $d l t A$ and $d l t D$ came from the collection of mutant strains created in the Quivey laboratory (Quivey et al. 2015). In addition, a strain defective in $g t f B$ was used as control, because it has impaired biofilm formation in vitro (Koo et al. 2010; Xiao et al. 2012), and reduced pathogenicity in vivo (Yamashita et al. 1993). The gtfB mutant was kindly provided by Dr Robert A. Burne (Department of Oral Biology, University of Florida, Gainesville, Florida). Actinomyces naeslundii ATCC 12104 and Streptococcus gordonii DL-1 were also used in experiments examining mixed-species biofilm formation. S. gordonii and $A$. naeslundii are early colonizers, while $S$. mutans is a proven virulent (cariogenic) oral pathogen. Further, S. gordonii strain DL-1 and A. naeslundii strain ATCC 12104 are also acidogenic and produce EPS, glucans or fructans, respectively (Haisman and Jenkinson 1991; Bergeron et al. 2000), thus contributing to ECM assembly and acidogenesis. In the mixed-species model, $S$. mutans is the cariogenic organism, $S$. gordonii is a representative of a commensal, early colonizer, non-mutans streptococci that binds salivary a-amylase, and A. naeslundii is a representative of an early colonizer non-streptococci (Takahashi and Nyvad 2011). Strains were stored at $-80^{\circ} \mathrm{C}$ in tryptic soy broth containing $20 \%$ glycerol, and were plated on blood agar plates or BHI agar plates with appropriate antibiotics (see Table 1).

\section{Biofilm preparation}

The single- and mixed-species biofilms were formed on HA disks (1.25 cm diameter, Clarkson Chromatography Products, Inc., South Williamsport, PA, USA) coated with filter-sterilized, clarified human whole saliva (sHA). Saliva and pellicle preparation were performed as described by Lemos et al. (2010). Four volunteers were chosen to donate saliva who had not used antimicrobial treatments in the last three months (two males and two females, 20-38 years old). Each volunteer was asked to rinse their mouth with $5 \mathrm{ml}$ of ultra-pure water, then masticate a piece of parafilm, collecting $5 \mathrm{ml}$ saliva into a collection tube, which was then discarded. Next, the volunteers continued masticating the parafilm and collected saliva into an ice-chilled tube. The saliva samples from all volunteers were pooled and diluted 1:1 with adsorption buffer $(\mathrm{AB}$ buffer: $50 \mathrm{mM} \mathrm{KCl}, 1 \mathrm{mM} \mathrm{KPO}, 1 \mathrm{mM} \mathrm{CaCl}_{2}, 1 \mathrm{mM}$ $\mathrm{MgCl}_{2}, 0.1 \mathrm{mM}$ PMSF, in dd- $\mathrm{H}_{2} \mathrm{O}, \mathrm{pH}$ 6.5). Saliva was centrifuged $(5,000 \times \mathrm{g} / 10 \mathrm{~min})$ and the clarified portion was filtered sterilized $(0.22 \mu \mathrm{m}$ low protein binding polyethersulfone membrane filter). Saliva was used fresh for pellicle formation and medium preparation at the start of the experiment, and any remaining saliva was aliquoted and stored at $-80^{\circ} \mathrm{C}$ until use for culture medium dilution. Alpha amylase activity was measured using the Salivary Alpha-Amylase Assay Kit (Salimetrics, State College, PA, USA), with both fresh and thawed samples having activity $\sim 24$ units $\mathrm{ml}^{-1}$. Saliva-coated HA disks were placed in a vertical position in a 24 -well microtiter dish using a custom-made disk holder (Koo et al. 2010; Figure S1).

S. mutans parental strain (UA159) or deletion strains, A. naeslundii ATCC 12104 and S. gordonii DL-1 cells were grown in tryptone-yeast extract broth (TY; $2.5 \%$ tryptone, $1.5 \%$ yeast extract) containing $1 \%$ glucose at $37^{\circ} \mathrm{C}$ in a $5 \% \mathrm{CO}_{2} / 95 \%$ air atmosphere to late-exponential phase $\left(\mathrm{OD}_{600 \mathrm{~nm}} 1.0\right.$ for streptococci and $\mathrm{OD}_{600 \mathrm{~nm}}$ 1.5 for A. naeslundii). For mixed-species biofilms, each bacterial suspension was mixed to provide an inoculum with a defined microbial population of $S$. mutans, $A$. naeslundii, and S. gordonii of $10^{6}$ colony-forming units $\left(\mathrm{CFU} \mathrm{ml} \mathrm{m}^{-1}\right)$. Single-species biofilms consisted of only $S$. mutans $\left(10^{6} \mathrm{CFU} \mathrm{ml}{ }^{-1}\right)$ strains. The mixed population of $S$. mutans, A. naeslundii plus $S$. gordonii and the single-species (S. mutans only) were inoculated in $2.8 \mathrm{ml}$ of TY with $0.1 \%$ sucrose and $25 \%$ saliva, and incubated at $37^{\circ} \mathrm{C}$ in a $5 \% \mathrm{CO}_{2} / 95 \%$ air atmosphere. The concentration of sucrose and starch was based on previous experiments with S. mutans single-species biofilms (Klein et al. 2010).

During the first $19 \mathrm{~h}$, the organisms were grown undisturbed to allow initial biofilm formation. At $19 \mathrm{~h}$, the culture medium was replaced by transferring the custom-made disk holder with nascent biofilms to wells containing fresh medium (TY $+0.1 \%$ sucrose and $25 \%$ saliva) using forceps. After $29 \mathrm{~h}$ of biofilm growth, both mixed-species and S. mutans biofilms were transferred to TY containing $0.5 \%$ sucrose, $1 \%$ starch and $25 \%$ saliva to increase the amount of carbohydrate to serve as substrate for microbial metabolism and matrix build-up. The culture medium was then changed twice daily ( 8 am and $6 \mathrm{pm}$ ) until the end of the experimental period (Figure S1). Thus, the biofilms were kept immersed in $0.1 \%$ sucrose $/ 25 \%$ saliva for $10 \mathrm{~h}$, and in $0.5 \%$ sucrose $/ 1 \%$ starch $/ 25 \%$ saliva for $14 \mathrm{~h}$. This scenario mimics what may be observed in dental niches that retain food after eating. After each medium change, the $\mathrm{pH}$ of the spent medium was measured. The TY medium $+0.1 \%$ sucrose $/ 25 \%$ saliva was prepared by diluting $1.25 \times$ TY with saliva in a $1: 4$ ratio, resulting in $1 \times \mathrm{TY}$, to which an appropriate volume of sucrose was added to achieve a final concentration of $0.1 \%$. TY containing $0.5 \%$ sucrose $/ 1 \%$ starch $/ 25 \%$ saliva was prepared by diluting $1.25 \times$ TY containing $1.25 \%$ starch with saliva in a $1: 4$ ratio, resulting in $1 \times$ TY containing $1 \%$ starch, to which an appropriate volume of sucrose was added to achieve a final concentration of $0.5 \%$. Addition of 
saliva to the culture medium was performed immediately before use to a final concentration of $25 \%$ [based on a previous study (Rogers et al. 2001)], and served two purposes: to provide starch hydrolysates to the culture medium via hydrolyzation by salivary amylase and to supply substrates (urea and arginine) for alkali production by S. gordonii and A. naeslundii (Jakubovics et al. 2008; Liu et al. 2012; Nascimento et al. 2013).

\section{Biofilm analyses}

\section{Determination of microbial population, biomass and} the biochemical characteristics of biofilm ECM

The sHA disks containing biofilm cultures were removed and processed for population, biomass, and biochemical assays at distinct phases of development to provide an overall characterization of biofilms formed by the mutant strains of $S$. mutans. For microbial population evaluation, the mixed-species biofilms were processed at distinct time points (Figure S1). S. mutans single-species biofilms were evaluated at 67 and $115 \mathrm{~h}$. Biomass quantification and biochemical assays were performed using 67 and 115 h-old mixed- and single-species biofilms to determine the content of eDNA (Rice et al. 2007), LTA (described below), soluble and insoluble EPS (Lemos et al. 2010), and proteins (in the soluble and insoluble portion of biofilm suspensions).

At the selected ages, biofilms were dip-washed into wells containing sterile $0.89 \% \mathrm{NaCl}$ solution (saline solution). Each biofilm (disk) was transferred to a glass tube containing $1 \mathrm{ml}$ of saline solution. Next, $1 \mathrm{ml}$ of saline solution was used to wash the walls of each tube. The glass tubes with biofilms/disks were placed in a beaker and subjected to water-bath sonication for $10 \mathrm{~min}$. A sterile metal spatula was used to scrape off any remaining biofilm from each disk surface, and the $2 \mathrm{ml}$ of each biofilm suspension were transferred to a new $15 \mathrm{ml}$ tube. Next, each glass tube was washed with $3 \mathrm{ml}$ of saline solution, which was transferred to the tube containing the initial $2 \mathrm{ml}$, yielding $5 \mathrm{ml}$ of total biofilm suspension per biofilm/disk. Each biofilm suspension $(5 \mathrm{ml})$ was sonicated using a probe at $7 \mathrm{~W}$ for $30 \mathrm{~s}$.

An aliquot of each suspension $(0.1 \mathrm{ml})$ was used for a 10 -fold serial dilution to determine the number of colony forming units (CFU) by plating onto blood agar plates (incubated in a $5 \% \mathrm{CO}_{2} / 95 \%$ air atmosphere, $37^{\circ} \mathrm{C}, 48 \mathrm{~h}$ ). The colony morphology of each species was visually differentiated (Figure S1). The remaining volume $(4.8 \mathrm{ml})$ was centrifuged $\left(5,500 \times g / 10 \mathrm{~min} / 4^{\circ} \mathrm{C}\right)$. The supernatant (with soluble ECM components) was transferred to a new tube and the pellet (precipitate with the microbial cells and insoluble ECM components) was washed twice with $2.6 \mathrm{ml}$ of sterile Milli- $\mathrm{Q}$ water $\left(5,500 \times g / 10 \mathrm{~min} / 4^{\circ} \mathrm{C}\right)$. The supernatants generated during the two washes were combined with the first supernatant obtained, totaling $10 \mathrm{ml}$, which was used to isolate and quantify water-soluble polysaccharides (WSP; Dubois et al. 1956), eDNA (Rice et al. 2007), LTA (described below), and protein (Bradford, 1976). The pellet was suspended in $2.55 \mathrm{ml}$ of Milli-Q water, of which $0.5 \mathrm{ml}$ was used for quantification of insoluble dry-weight (biomass), $0.05 \mathrm{ml}$ for the quantification of total protein (Bradford 1976) and $1 \mathrm{ml}$ for the isolation and quantitation of water-insoluble polysaccharides (or alkali soluble polysaccharide - ASP; Dubois et al. 1956).

Isolation and quantification of LTA. The isolation of LTA from S. mutans UA159 for standard curve construction was performed following the methodology described by Sutcliffe and Hogg (1993). To isolate LTA from ECM, a $3 \mathrm{ml}$ aliquot of supernatant (soluble portion) recovered during biofilm processing (above) was mixed with $150 \mu \mathrm{l}$ of trichloroacetic acid (TCA, Sigma-Aldrich, St. Louis, MO, USA) to a final concentration of $5 \%$. These samples were incubated with agitation at $22^{\circ} \mathrm{C}$ for $18 \mathrm{~h}$. Next, samples were centrifuged $(6,500 \times g / 30 \mathrm{~min})$, the supernatant was collected (saved) and an aliquot of $6 \mathrm{ml}$ of ethyl ether (Sigma-Aldrich) was used to suspend the pellet, followed by centrifugation $(6,500 \times g / 30 \mathrm{~min})$. The resulting supernatant was mixed with the initial supernatant, to which three volumes of acetone were added, followed by incubation at $4^{\circ} \mathrm{C}$. After $24 \mathrm{~h}$, the samples were centrifuged $(6,500 \times g / 30 \mathrm{~min})$, and the pellet washed three times with $5 \mathrm{ml}$ of acetone. The pellets were air-dried, and suspended in $100 \mu \mathrm{l}$ of MilliQ water. These samples were stored at $-80^{\circ} \mathrm{C}$, until quantification via indirect ELISA.

Briefly, the antigens (LTA samples from either the standard curve or derived from the biofilm matrix) were used to coat microplates (at $4^{\circ} \mathrm{C}$ for $24 \mathrm{~h}$ ). Uncoated LTA was removed with three washes of $200 \mu \mathrm{l} 1 \times \mathrm{PBS}(137 \mathrm{mM}$ $\mathrm{NaCl}, 2.7 \mathrm{mM} \mathrm{KCl}, 8 \mathrm{mM} \mathrm{Na}_{2} \mathrm{HPO}_{4}$, and $2 \mathrm{mM} \mathrm{KH}_{2} \mathrm{PO}_{4}$, $\mathrm{pH} 7$ ) containing $0.2 \%$ Tween 20 (PBST) per well, and wells were blocked with $200 \mu \mathrm{l}$ of $2 \%$ bovine serum albumin (BSA). Plates were incubated for $2 \mathrm{~h}$ at room temperature. The plates were washed twice with PBST, and $100 \mu \mathrm{l}$ of primary antibody (Gram positive bacteria LTA antibody, Thermo Fisher Scientific, Rockford, IL, USA; diluted 1:200) were added, followed by incubation at $4^{\circ} \mathrm{C}$ for $18 \mathrm{~h}$. The plates were then washed four times with PBST. Next, $100 \mu \mathrm{l}$ of conjugated secondary antibody [goat anti-mouse IgG $(\mathrm{H}+\mathrm{L}), \mathrm{HRP}$ conjugate, Invitrogen; diluted 1:2,000] were added to each well, with incubation for $2 \mathrm{~h}$ at room temperature. Subsequently, the plates were washed four times with PBST. Finally, $100 \mu \mathrm{l}$ of $30 \%$ hydrogen peroxide were added to each well, the plates were incubated at room temperature for $4 \mathrm{~h}$, and the absorbance was measured at $405 \mathrm{~nm}$ on an ELISA plate reader (Biochrom Ez read 400, Cambourne, UK). 
Glycosyl linkage analysis of the water-soluble and -insoluble exopolysaccharides in the ECM of mixed-species biofilms. The structure of the extracellular water-soluble and -insoluble polysaccharides was determined by linkage analysis using the partially methylated alditol acetates (PMAA) derivatization method for gas chromatography mass spectrometry (GC-MS). Briefly, mixed-species biofilms comprised of $S$. mutans UA159 parental and mutant strains were grown as described earlier, and subjected to WSP and ASP separation. Mixed-species biofilms of each $S$. mutans strain were prepared for three distinct experiments (four replicates per experiment) and pooled together, per strain, to obtain a minimum of $3 \mathrm{mg}$ per sample. Samples ( $3 \mathrm{mg}$ ) were dissolved in $500 \mu \mathrm{l}$ of dimethyl sulfoxide (DMSO) to which $200 \mu \mathrm{l}$ of n-butyl lithium were added, and tubes were incubated for $40 \mathrm{~min}$ at room temperature. Methyl iodide (300 $\mu \mathrm{l})$ was added and stirred for $1 \mathrm{~h}$. The resulting mixtures were partitioned with deionized water and chloroform. The chloroform layer was separated and dried under a stream of nitrogen. Samples were hydrolyzed with $2 \mathrm{M}$ trifluoroacetic acid for $1 \mathrm{~h}$ at $121^{\circ} \mathrm{C}$ containing $1 \mathrm{mg} \mathrm{ml}^{-1}$ of inositol as an internal standard. The samples were then dried under a stream of nitrogen. Dry samples were reduced with $100 \mu \mathrm{l}$ of $1 \mathrm{M}$ ammonium hydroxide with $20 \mathrm{mg} \mathrm{ml}^{-1}$ of sodium borodeuteride in DMSO for $90 \mathrm{~min}$ at $40^{\circ} \mathrm{C}$. The reaction was quenched with glacial acetic acid. Samples were O-acetylated with $100 \mu \mathrm{l}$ of 1-methylimidazole and $500 \mu \mathrm{l}$ of acetic anhydride. The resulting partially methylated alditol acetates were partitioned with deionized water and methylene chloride. The methylene chloride layer was separated and dried under a stream of nitrogen. Samples were dissolved in $1 \mathrm{ml}$ of acetone and analyzed by GC-MS (GC: Agilent 7890A (Santa Clara, CA, USA) equipped with FID detector, MS: Agilent 5975C, column SP-2330). Linkage points were identified by comparing peak mass fragmentation with the known mass fragmentation in the library.

\section{Laser scanning confocal fluorescence microscopy} imaging of biofilms and computational analyses

The 3-D morphology and structural organization of the single- and mixed-species biofilms were examined by simultaneous in situ labeling of EPS and bacterial cells, as previously described (Klein et al. 2009). Briefly, $1 \mu \mathrm{M}$ Alexa Fluor 647 -labeled dextran conjugate (absorbance/ fluorescence emission maxima of 647/668 nm; Molecular Probes, Carlsbad, CA, USA) was added to the culture medium at the beginning of, and during, development of the biofilms. Bacteria in the biofilms were labeled using SYTO $^{\circ}$ green fluorescent nucleic acid stain $(485 / 498 \mathrm{~nm}$; Molecular Probes) using standard protocols (Klein et al. 2009). The imaging was performed using a Zeiss LSM 780 microscope (Zeiss, Jena, Germany) equipped with a
$20 \times$ objective lens. Each biofilm was scanned at four randomly selected positions, and a series of confocal images were generated by optical sectioning at each of these positions. The confocal images were analyzed using Amira 6.0.1 (Mercury Computer Systems Inc., Chelmsford, MS, USA) to create three-dimensional renderings of each structural component (EPS and bacteria) of the biofilms for visualization of the morphology and 3-D architecture as detailed previously (Xiao et al. 2012). Moreover, quantification of total bacterial content and EPS matrix was performed using COMSTAT version 2 (Heydorn et al. 2000; https://www.comstat.dk).

\section{Statistical analyses}

The data were analyzed to examine whether there were differences between the biofilms formed by each mutant strain and the parental strain of $S$. mutans in single- and mixed-species biofilms, at distinct developmental phases using Prism 7 software (GraphPad Software, Inc., La Jolla, $\mathrm{CA}$, USA). The $\mathrm{pH}$ values of the spent media were evaluated by two-way ANOVA using as factors biofilm type (single- $v s$ mixed-species) and biofilm developmental phase (time), followed by Tukey's test; $p<0.05$ ). Data from population, biomass and biochemical analyses were evaluated by two-way ANOVA using as factors matrix component modulation by $S$. mutans strains (parental strain and deletion strains), and biofilm developmental phase (67 h- and $115 \mathrm{~h}$-old biofilms) in two types of biofilms, followed by either Tukey's multiple comparison test or Sidak's multiple comparison test $(\alpha=0.05)$. Moreover, Pearson's correlation analyses were performed between $S$. mutans (or total) population, biomass, protein, and matrix components (eDNA, LTA, WSP, ASP and proteins) for each $S$. mutans strain (parental strain and deletion strains). Biovolume quantification of the bacteria and EPS in single- and mixed species biofilms from confocal microscopy were evaluated by two-way ANOVA using as factors distinct strains and type of biofilm component (bacteria vs EPS).

\section{Results}

The eDNA, LTA, soluble and insoluble EPS, and proteins of the extracellular matrix derived from single-species biofilms (S. mutans UA159 (parental strain) or the deletion strains) and mixed-species biofilms (S. mutans UA159 or mutant strains, A. naeslundii and S. gordonii) were evaluated. The glycosyl linkages of soluble and insoluble EPS were determined for mixed-species biofilms. In addition, the $\mathrm{pH}$ of the spent culture medium, the microbial population and the biomass of biofilms were assessed. Lastly, the 3-D structure of the biofilms, including the matrix and microbial content, were examined. Overall, distinct 
features were observed depending on the gene that was inactivated and on the type of biofilm culture.

\section{Overall features of single- and mixed-species biofilms}

\section{pH of the spent culture medium}

The $\mathrm{pH}$ values of spent culture medium from single- and mixed-species biofilms were evaluated during the development of these biofilms at 19, 29, 43, 53, 67, 77, 91, 101, and $115 \mathrm{~h}$ (Figure $1 \mathrm{~A}$ and $\mathrm{B}$, respectively). The $\mathrm{pH}$ values at $43,67,91$ and $115 \mathrm{~h}$ were significantly lower when compared to the $\mathrm{pH}$ values found at $19 \mathrm{~h}$ for single- and mixed-species biofilms. At those four times, the $\mathrm{pH}$ values were, on average, below 5.5. The presence of the dietary carbohydrates ( $0.5 \%$ sucrose and $1 \%$ starch) in the culture medium mimicked a cariogenic challenge, as these carbohydrates would be metabolized into acids (and exopolysaccharides). Further, the lowest $\mathrm{pH}$ values for the spent medium were detected in all single-species biofilms at 29, 53 and $77 \mathrm{~h}$ ( $v s$ mixed-species biofilms, $p \leq 0.05$ ).

\section{Population dynamics at different developmental phases of mixed-species biofilm}

A longitudinal analysis was performed to examine how the three species, $S$. mutans (either parent strain or a deletion strain), S. gordonii, and A. naeslundii, grew as the mixed-biofilms developed, and to determine whether the absence of a specific gene affected the population dynamics. The three species were detected at all ages of biofilms formed with the $S$. mutans parental strain or the deletion strains, except at $115 \mathrm{~h}$, when $A$. naeslundii was not detected (Figure $2 \mathrm{~A}-\mathrm{F}$ ). The population dynamics show that $S$. gordonii was the most prevalent species at the early stages (29-53 h) of biofilm formation, consistent with its role as an early colonizer. However, after $67 \mathrm{~h}$ there was a steady increase in the numbers of $S$. mutans, regardless of the gene deletions tested in this study, with a concomitant reduction in $S$. gordonii and a substantial decrease in $A$. naeslundii (Figure 2). Thus, in the in vitro model used here, $67 \mathrm{~h}$ could be considered a transitional phase, key to biofilm development and maturation.

\section{Bacterial population of mixed-and single-species biofilms at 67 and $115 \mathrm{~h}$}

At $67 \mathrm{~h}$, mixed-species biofilms formed with any of the deletion strains showed a similar behavior compared to the parental strain UA159 (Figure 3A): there was a small, not statistically significant, increase in $S$. gordonii. At this stage, S. mutans was not predominant, but with time it become the predominant species in the population, as seen in $115 \mathrm{~h}$-old mixed-species biofilms (Figure 3B). For $115 \mathrm{~h}$-old mixed-species biofilms, there was a significant increase in $S$. mutans species for all five mutant strains ( $\Delta g t f B, \Delta l y t T, \Delta l y t S$, $\Delta d l t A$ and $\Delta d l t D)$ and the parental strain, with the largest $\mathrm{CFU} / \mathrm{biofilm}$ detected for the $\Delta g t f B$ strain $(p<0.05)$. Of note, A. naeslundii was not detected at $115 \mathrm{~h}$ (see also Figure 2).

In single-species biofilms, a greater population of $\triangle g t f B$ was found at 67 and $115 \mathrm{~h}$ (Figure 3C). In addition, there was a significant difference in the CFU values detected for $\Delta g t f B$ compared to all other strains, except $\Delta d l t D$, in $115 \mathrm{~h}$-old $S$. mutans single-species biofilms $(p<0.05$; Figure $3 C$ ). Nevertheless, the $\Delta g t f B$ strain was more abundant when compared to the other strains at $115 \mathrm{~h}$. In addition, there was a significant decrease in the CFU recovered from mixed-species biofilms containing the $\Delta l y t T$ strain at $115 \mathrm{~h}$, compared to $67 \mathrm{~h}$ (Figure 3C).

\section{Biomass and protein content in the insoluble portion of biofilms}

The insoluble biomass data for 67 and $115 \mathrm{~h}$-old biofilms are shown in Figure 4A and B, respectively. No differences were found in the biomass in single- (Figure 4A) and mixed-species (Figure 4B) biofilms at both ages between
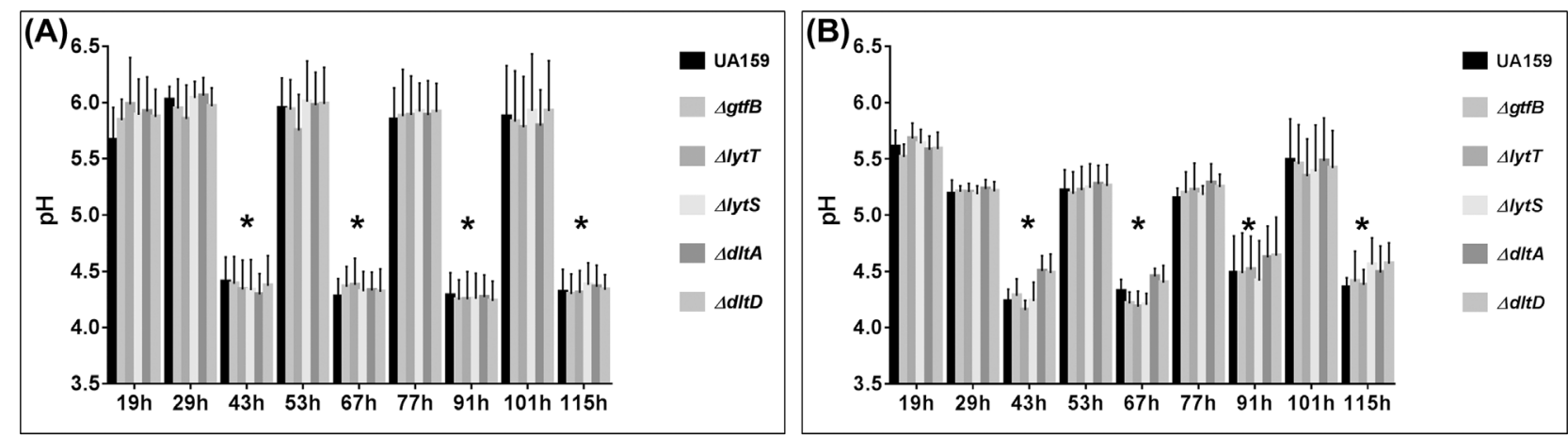

Figure 1. pH measurements from single- and mixed-species biofilm cultures.

Notes: $\mathrm{pH}$ values for (A) single- and (B) mixed-species biofilms. Biofilms were evaluated at 19, 29, 43,53,67,77,91, 101 and $115 \mathrm{~h}$ by measuring the $\mathrm{pH}$ values of the spent medium. Statistically significant differences in $\mathrm{pH}$ were observed at $43,67,91$ and $115 \mathrm{~h}$ for the $\mathrm{S}$. mutans parental strain and all mutant strains of $S$. mutans, tested in both single- and mixed-species biofilms (* two-way ANOVA using as factors biofilm type (single- $v s$ mixed-species) and biofilm developmental phase (time), followed by Tukey's test; $p<0.05)$. The data shown are averages and error bars indicate the SDs $(n=6$ for all time points except for $67 \mathrm{~h}$, where $n=10)$. 

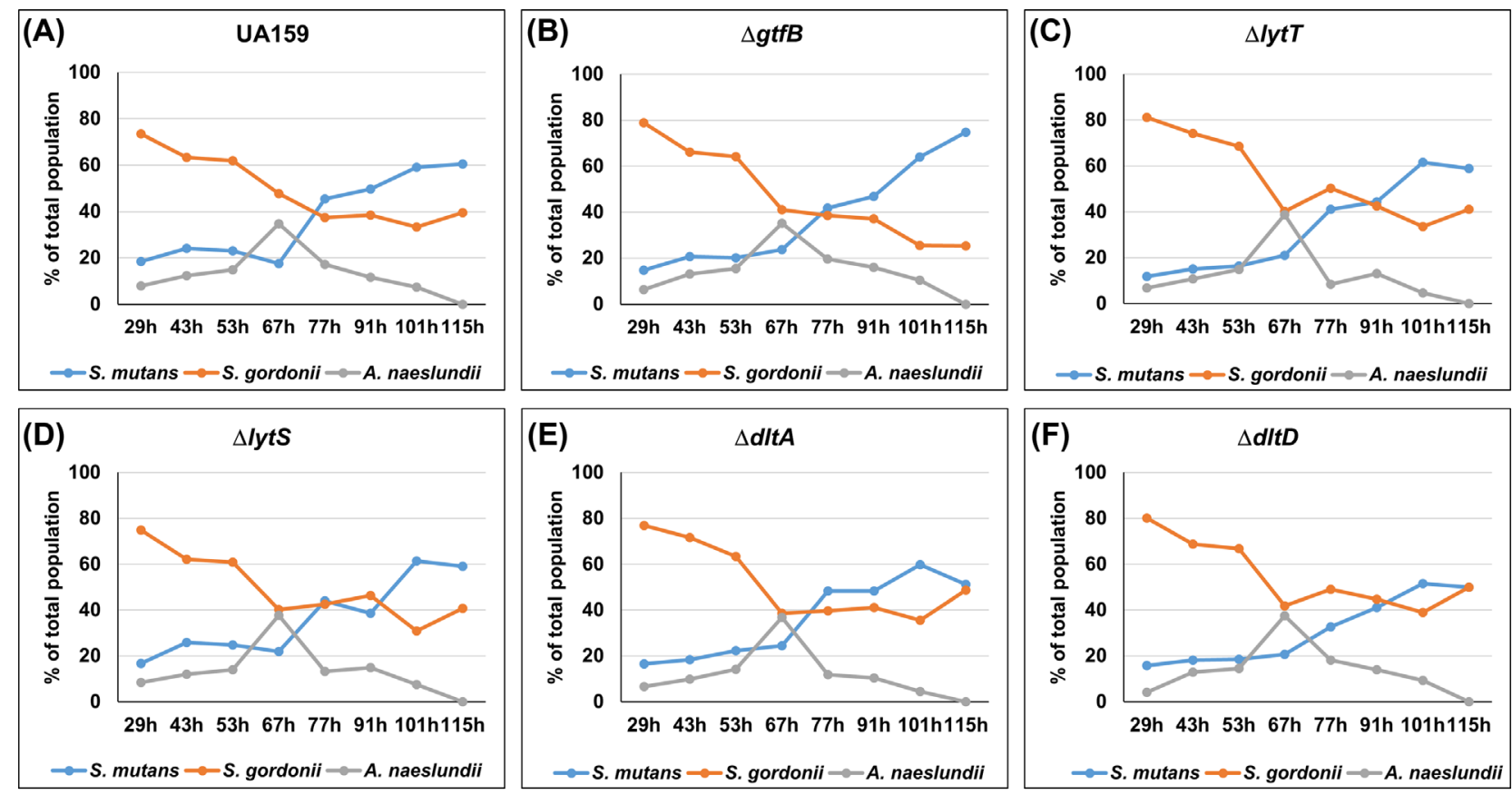

Figure 2. Population dynamics of S. mutans, S. gordonii and A. naes/undii species during the formation of mixed-species biofilms. Notes: The data shown are average proportions of all species at $29,43,53,67,77,91,101$, and $115 \mathrm{~h}$ post-inoculation of the biofilm cultures. Data are represented as the percentage total population for each strain: S. mutans (blue line), S. gordonii (orange line), and A. naeslundii (gray line), where in each panel the S. mutans strain is: (A) UA159 parental strain; (B) $\Delta g t f B ;$ (C) $\Delta l y t T$; (D) $\Delta l y t S$; (E) $\Delta d l t A$; and (F) $\Delta d l t D$. The data shown are averages ( $n=6$ for all time points, except for $67 \mathrm{~h}$, where $n=10)$.

different strains of S. mutans. However, there was a progressive increase in the biomass of all biofilms from 67 to $115 \mathrm{~h}$, and this increase may be related to the increase in specific components of the ECM over time, further discussed below.

The protein content in the insoluble portion of singleand mixed-species biofilms at $67 \mathrm{~h}$ did not differ between S. mutans mutant strains and the parental strain (Figure $4 \mathrm{C}$ and D). Single-species biofilms formed by $\Delta g t f B$ contained a lower amount of total protein compared to single-species biofilms formed by the $\Delta l y t T, \Delta d l t A$ and $\Delta d l t D$ strains at $115 \mathrm{~h}$ (Figure $4 \mathrm{C}$ ). Further, mixed-species biofilms containing the $\Delta g t f B$ strain possessed a lower protein content than biofilms formed by the strains $\Delta d l t A$ and $\Delta d l t D$ at age $115 \mathrm{~h}(p<0.05)$ (Figure $4 \mathrm{D})$.

\section{The extracellular matrix components in single- and mixed-species biofilms}

\section{eDNA}

The amount of eDNA recovered from the soluble ECM portion of single- and mixed-species biofilms is depicted in Figure 5A and $\mathrm{B}$, respectively. Comparisons of the amounts of eDNA detected at 67 and $115 \mathrm{~h}$ demonstrated a decrease for the parental strain UA159 and an increase for $\Delta g t f B$ in single-species biofilms (Figure $5 \mathrm{~A}$ ), while for mixed-species biofilms only those formed with the $\Delta l y t S$ and $\Delta l y t T$ strains showed a significant increase over time $(p<0.05)$ (Figure 5B).
In single-species biofilms, at $67 \mathrm{~h}$, the parental strain, $S$. mutans UA159, yielded similar amounts of eDNA as were observed with the $\Delta l y t S$ and $\Delta l y t T$ strains $(p>0.05)$, while at $115 \mathrm{~h}, \Delta l y t S$ and $\Delta l y t T$ biofilms contained a greater amount of eDNA. For mixed-species biofilms at $67 \mathrm{~h}$ there were no statistical differences in the amount of eDNA recovered $(p>0.05)$, although eDNA was proportional to the total number of bacteria [except in the case of the $\Delta g t f B$ strain, as correlation analysis of eDNA quantity and total population were negatively correlated in this strain $(r=-0.8471 ; p=0.0701)]$. The similarity in the amount of eDNA detected in mixed-species biofilms may be due to the contribution of eDNA from the other species (S. gordonii and A. naeslundii). However, at $115 \mathrm{~h}$, a significantly higher amount of eDNA was measured in mixed-species biofilms containing $\Delta l y t S$ and $\Delta l y t T$, compared to all other deletion strains tested in mixed-species biofilms $(p<0.05)$ (Figure 5B), but correlation analysis demonstrated that the eDNA was not proportional to the total number of bacteria for UA159 $(r=0.8419 ; p=0.0099)$; $\Delta g t f B(r=-0.6588$; $p=0.1548) ; \Delta \operatorname{lyt} T(r=-0.9447 ; p=0.0045)$.

\section{Extracellular lipoteichoic acids}

LTA were detected in the matrix of all single- and mixed-species biofilms, using the methodology developed in this study (Figure 5C and D, respectively). Overall, the amount of LTA measured at 67 and $115 \mathrm{~h}$ increased as the biofilms aged; however, the values were statistically significant 

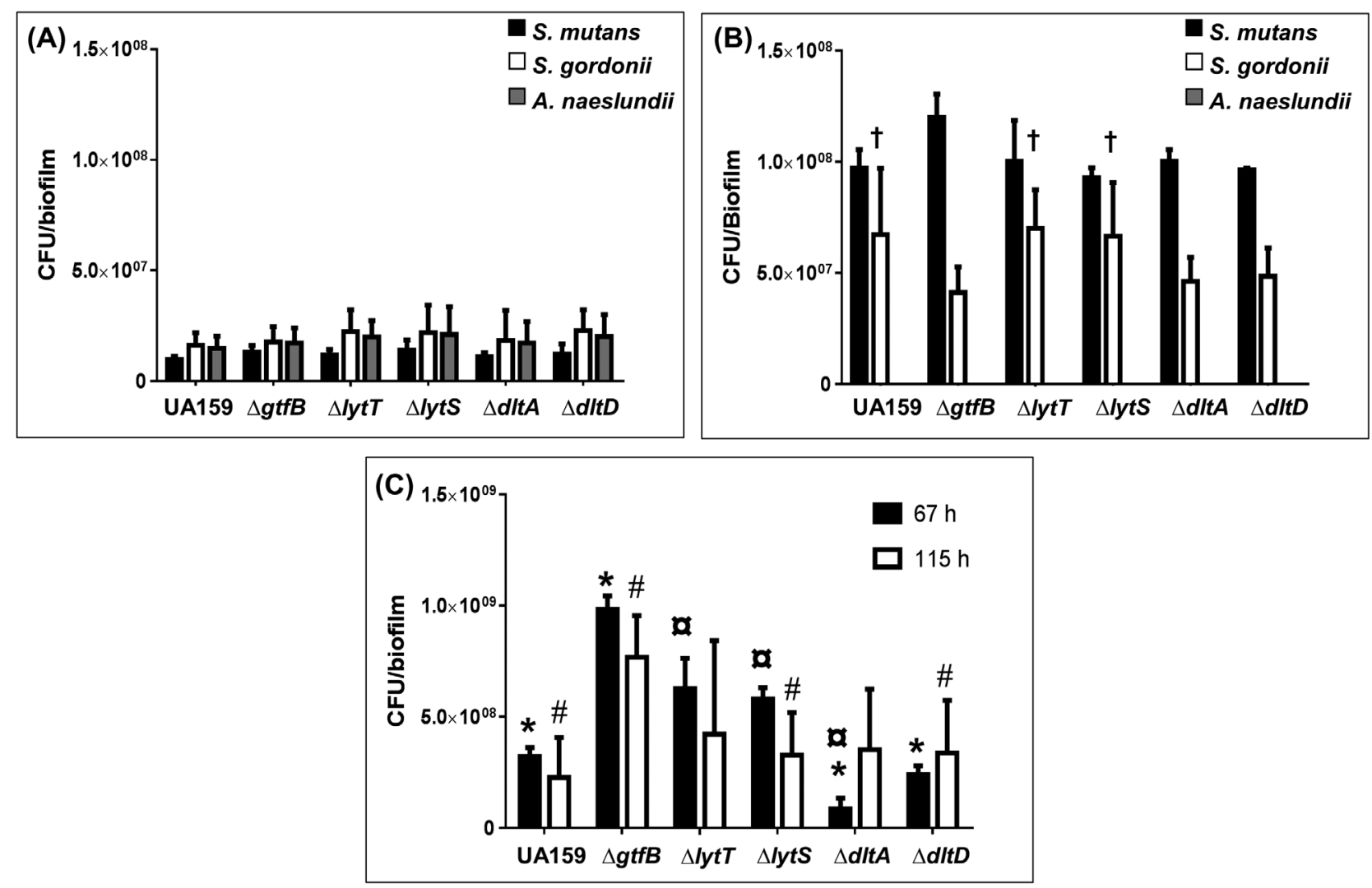

Figure 3. Bacterial population in single- and mixed-species biofilms at $67 \mathrm{~h}$ and $115 \mathrm{~h}$ development.

Notes: CFU detected in mixed-species biofilms after incubation for $67 \mathrm{~h}$ (Panel A) and $115 \mathrm{~h}$ (Panel B). No statistical differences in CFU values between strains were detected in the $67 \mathrm{~h}$ biofilm cultures (Panel A) ( $p>0.05$; two-way ANOVA using bacterial species and S. mutans strains as factors, followed by Tukey's test). In Panel B, aged $115 \mathrm{~h}$, CFU values are shown for the S. mutans strains and S. gordonii from mixed-species biofilms, as A. naes/undii was not detected. S. mutans was statistically the major inhabitant at $115 \mathrm{~h}$ in all mixed biofilms tested $(p>0.05$; two-way ANOVA using bacteria species and $S$. mutans strains as factors, followed by Tukey's test). The $S$. mutans strain $\triangle g t f B$ was detected in significantly higher quantity, compared to the other strains tested $(p<0.05)$. All biofilms contained significantly increased CFU values for $S$. mutans at $115 \mathrm{~h}$, compared to biofilms at $67 \mathrm{~h}$ (Panel B vs Panel A) ( $p<0.05$; two-way ANOVA using developmental phase and S. mutans strains as factors, followed by Sidak's test). Elevated numbers of $S$. gordonii were observed only in biofilms formed with the $S$. mutans strains UA159, $\Delta l y t S$ and $\Delta / y t T\left({ }^{\dagger} p<0.05\right.$; two-way ANOVA with developmental phase and S. mutans strains as factors, followed by Sidak's test). (Panel C) S. mutans CFU values from single-species biofilm cultures, where the symbols indicate significantly different CFU values in pairwise comparisons $(p<0.05)$. The $\Delta l y t T$ strain was the only S. mutans strain tested in single-species biofilms that differed in CFU recovered at $115 \mathrm{~h}$, compared to $67 \mathrm{~h}$ ( $p=0.0205$; two-way ANOVA using developmental phase and S. mutans strains as factors, followed by Tukey's test). The data shown are averages and error bars indicate the SD ( $n=6$ for singlespecies biofilms at 67 and $115 \mathrm{~h}$, and for mixed-species biofilms at $115 \mathrm{~h} ; n=10$ for mixed-species biofilms at $67 \mathrm{~h}$ ).

only in single-species biofilms of S. mutans UA159 (Figure 5C), and in mixed-species biofilms containing UA159, or the deletion strains $\Delta l y t T$ and $\Delta$ dltA (Figure 5D). In single-species biofilms, LTA levels were significantly different between the $\Delta l y t T$ and $\Delta d l t D$ strains at $67 \mathrm{~h}$ (Figure $5 \mathrm{C}$ ); while at $115 \mathrm{~h}, \Delta d l t A$ contained elevated levels of LTA, compared to the $\Delta l y t S$ and $\Delta l y t T$ strains $(p<0.05)$ (Figure $5 \mathrm{D})$. The increased extracellular LTA in the ECM of biofilms formed by $\Delta d l t A$ and $\Delta d l t D$ was unexpected because the genes $d l t A$ and $d l t D$ are involved with LTA metabolism and their deletion was anticipated to reduce the amount of LTA in the ECM (see Discussion section).

\section{Water-soluble and -insoluble exopolysaccharides in the ECM}

The amounts of water-soluble (WSP; recovered from the soluble portion of biofilms during processing) and water-insoluble (ASP; extracted from the insoluble portion of biofilms) exopolysaccharides are shown in Figure 6. For both single- and mixed-species biofilms, there was a sharp and significant increase in the amount of WSP and ASP recovered from the ECM between 67 to $115 \mathrm{~h}(p<0.05)$. Lesser amounts of WSP were detected in single-species biofilms formed by $\Delta g t f B$ at both 67 and $115 \mathrm{~h}$, as compared to all other strains, except $\Delta l y t T$, at $67 \mathrm{~h}$ (Figure 6A). Moreover, the WSP content in the ECM of single-species biofilms formed by the $\Delta l y t T$ strain was reduced at both $67 \mathrm{~h}$ ( $v s$ strains $\Delta d l t A$ and $\Delta d l t D$; $p \leq 0.0278$ ), and at $115 \mathrm{~h}$, compared to the parental strain (Figure 6A). The amount of WSP in mixed-species biofilms did not vary significantly between the strains of $S$. mutans tested in this study at either time period evaluated (Figure 6B).

Measurement of the ASP content of the ECM from single-species biofilms of all $S$. mutans strains were not statistically different at the $67 \mathrm{~h}$ time point (Figure 6C). 

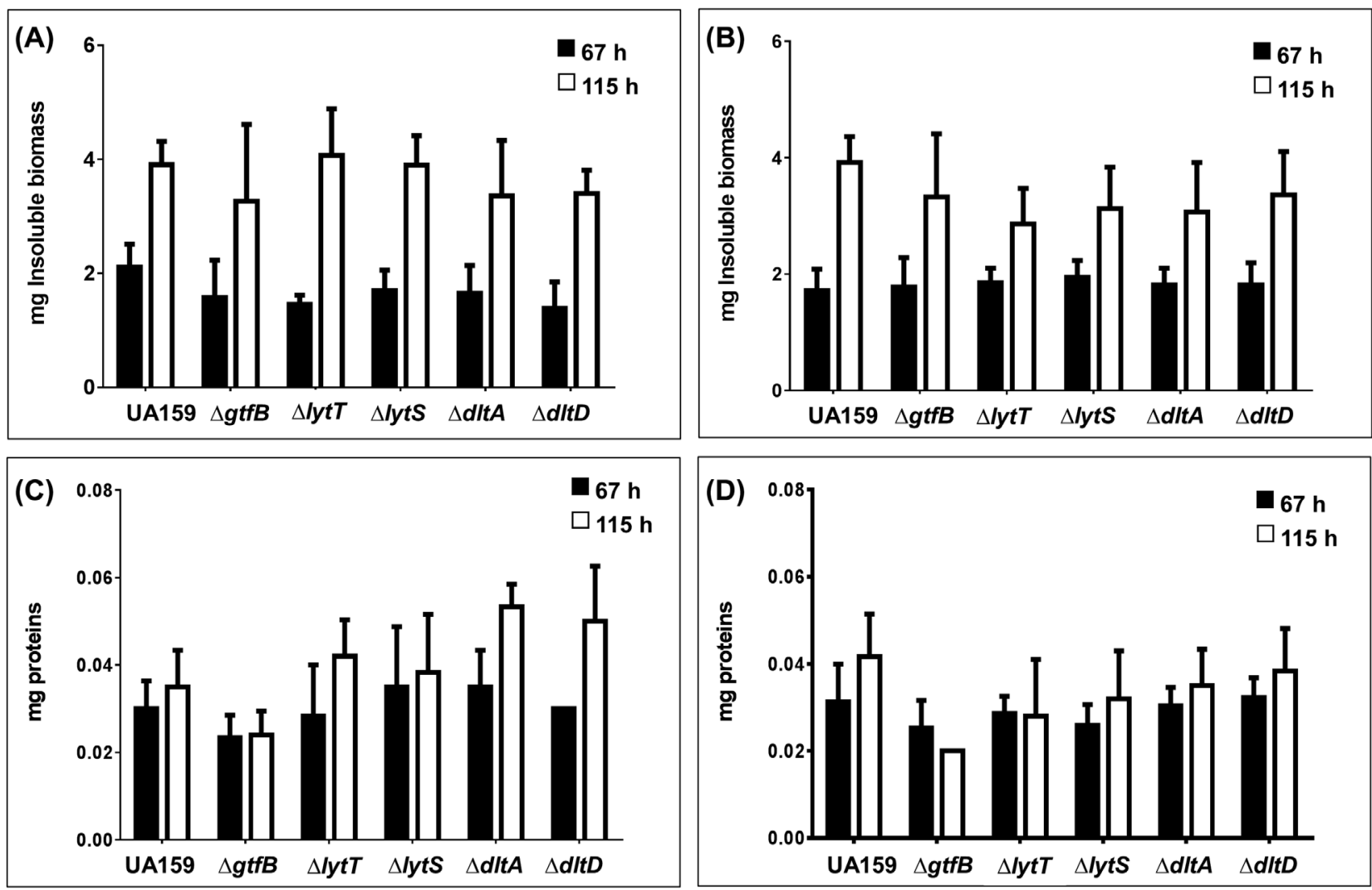

Figure 4. Insoluble biomass (dry-weight) and proteins from single- and mixed-species biofilms.

Notes: Insoluble biomass was measured from single- (Panel A) and mixed-species biofilms (Panel B) aged $67 \mathrm{~h}$ (black bars) and $115 \mathrm{~h}$ (white bars). A significant increase in insoluble biomass was observed for all strains between 67 and $115 \mathrm{~h}$ for both single- and mixed-species biofilms ( $p<0.05$; two-way ANOVA using developmental phase and S. mutans strains as factors, followed by Sidak's test). Total protein was determined from single- (Panel C) and mixed-species biofilms (Panel D) at 67 (black bars) and $115 \mathrm{~h}$ (white bars). At $67 \mathrm{~h}$, the protein content for both single- and mixed-species biofilms was not significantly different between tested strains. However, at $115 \mathrm{~h}, \Delta g t f B$ displayed a lower amount of protein in single- (vs $\Delta l y t T, \Delta d l t D$ and $\Delta d l t A$ ) and mixed-species biofilms (vs $\Delta d l t D$ and $\Delta d l t A$ ) $(p<0.05$, two-way ANOVA using developmental phase and $S$. mutans strains as factors, followed by Tukey's test). Moreover, there was a significant increase in protein content from 67 to $115 \mathrm{~h}$ for $\Delta d l t D$ and $\Delta d l t A$ in single-species biofilms $(p<0.05$; two-way ANOVA using developmental phase and $S$. mutans strains as factors, followed by Sidak's test). The plotted data are averages, and error bars indicate SDs ( $n=6$ for single-species biofilms at 67 and $115 \mathrm{~h}$, and for mixed-species biofilms at $115 \mathrm{~h} ; n=10$ for mixed-species biofilms at $67 \mathrm{~h}$ ).

At the $115 \mathrm{~h}$ time point, there was significantly less ASP measured in the $\Delta g t f B$ strain, compared to the $\Delta l y t T$ and $\Delta l y t S$ strains (Figure 6C). In addition, the amounts of ASP measured in mixed-species biofilms was similar among all tested strains at $67 \mathrm{~h}$; however, at $115 \mathrm{~h}$, a reduced amount of ASP was detected in biofilms formed by the $\triangle g t f B$ strain, compared to the parental strain UA159, or $\Delta d l t A$ and $\Delta d l t D$ (Figure 6D). Similarly, $\Delta l y t T$ yielded lower amounts of ASP, compared to the UA159 parental strain, in mixed-species biofilms at $115 \mathrm{~h}$.

Overall, there was a significant increase from 67 to $115 \mathrm{~h}$ in ASP and WSP in biofilms from all strains tested, in both single- and mixed-species cultures. The increase in the amounts of insoluble EPS can be directly related to the increase in total biomass for all S. mutans strains in single- and mixed-species biofilms, with the exceptions of $\Delta g t f B$ in single-species and $\Delta l y t T$ and $\Delta l y t S$ in mixed-species biofilms. Therefore, it is hypothesized that the lack of a functional lytST system may affect the synthesis of exopolysaccharides, especially in mixed-species biofilms.

In addition to measuring the amount of WSP and ASP, the glycosyl linkages by GC-MS were also examined. The type of linkages in ASP and WSP in mixed-species biofilms formed by the $S$. mutans strains are shown in Table 2. The glycosyl linkage of WSP from all biofilms were somewhat similar, except for $\Delta d l t D$. In the ASP, there was a lower percentage of 1,3-linked glucose in all mutant strains, compared to the parental strain, UA159. Moreover, the $\triangle d l t A$ strain only displayed 1,4-linked glucose. The ASP in the ECM of mixed-species formed by $S$. mutans UA159, $\Delta g t f B$ and $\Delta d l t D$ contained branched chains of glucose.

\section{Soluble proteins in the ECM}

The amounts of soluble proteins in the ECM of singleand mixed-species biofilms are shown in Figure S2. A 

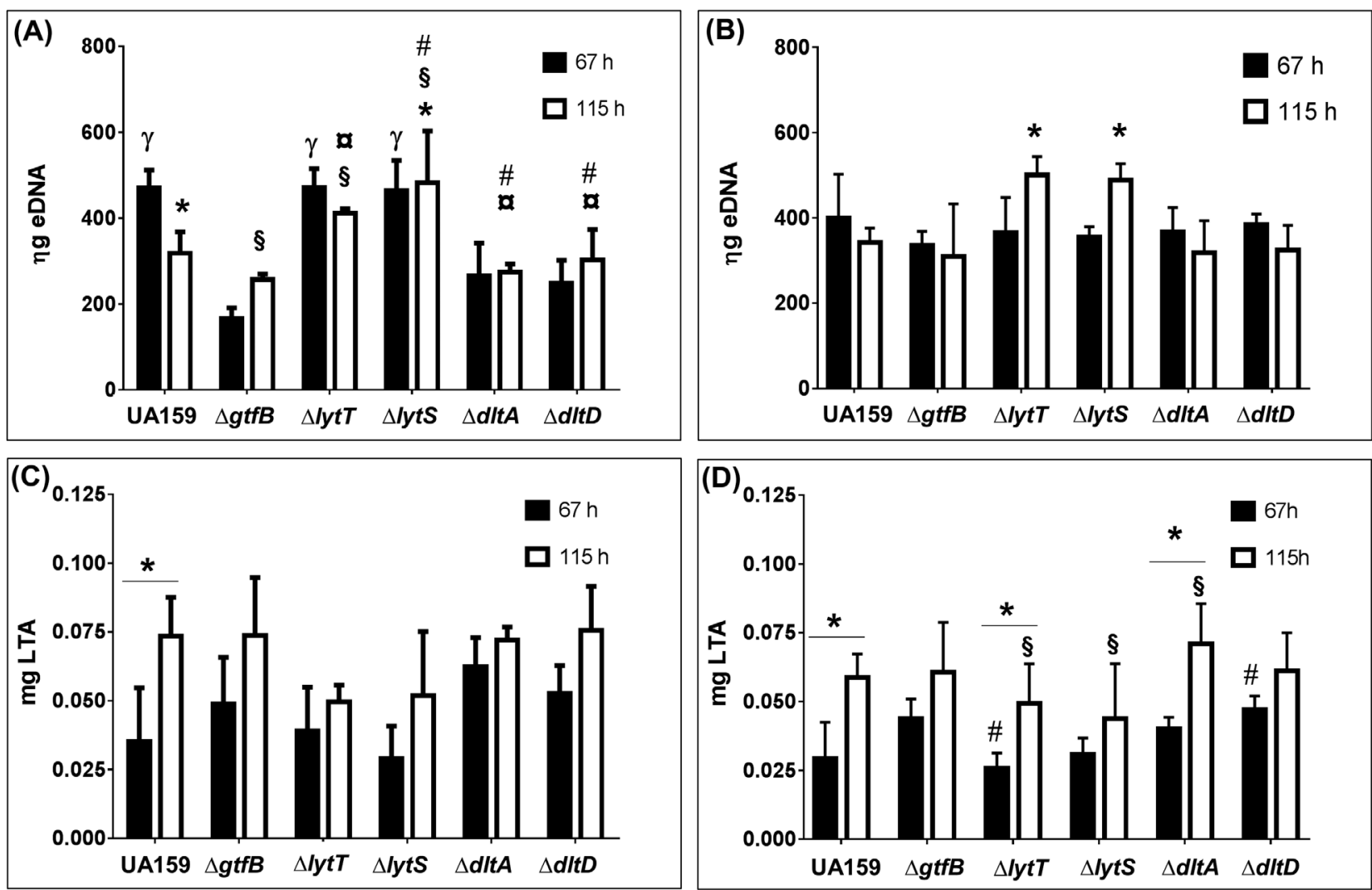

Figure 5. eDNA and LTA in the ECM of single- and mixed-species biofilms.

Notes: Total amounts of eDNA were measured from single- (panel A) and mixed-species (panel B) biofilms at $67 \mathrm{~h}$ (black bars) and $115 \mathrm{~h}$ (white bars). In singlespecies biofilms, at $67 \mathrm{~h}$, the parental strain UA159 yielded similar amounts of eDNA to those observed from the $\Delta / y t T$ and $\Delta / y t S$ strains ( $\left.{ }^{\prime} p>0.05\right)$, and quantities were greater than those measured from the other strains tested; while at $115 \mathrm{~h}, \Delta / y t T$ and $\Delta l y t S$ biofilms contained a greater amount of eDNA. Significant statistical differences are shown in comparisons of like symbols $\left({ }^{*} p<0.0001 ;{ }^{5} p \leq 0.0001 ; \not a p \leq 0.0141 ; \# p<0.0001\right.$; two-way ANOVA using developmental phase and $S$. mutans strains as factors, followed by Tukey's test). For mixed-species biofilms (Panel B), the amount of eDNA detected at $67 \mathrm{~h}$ was similar for all strains ( $p>0.05$ ), while at $115 \mathrm{~h}$, a significantly higher amount of eDNA was measured in biofilms with $\Delta l y t T$ and $\Delta l y t S$ compared to all other strains $\left(^{*} p<0.05\right.$; two-way ANOVA using developmental phase and S. mutans strains as factors, followed by Tukey's test). In addition, total amounts of extracellular LTA were measured from single(panel C) and mixed-species (panel D) biofilms at $67 \mathrm{~h}$ (black bars) and $115 \mathrm{~h}$ (white bars). Overall, the amount of LTA measured at 67 and $115 \mathrm{~h}$ increased as the biofilms aged; however, the values were statistically significant only in single-species biofilms of S. mutans UA159 (Panel C), and in mixed-species biofilms containing UA159, or the deletion strains $\Delta l y t T$ and $\Delta d l t A$ (Panel D) ${ }^{*} p<0.05$; two-way ANOVA using developmental phase and $S$. mutans strains as factors, followed by Sidak's test). In mixed-species biofilms, the amounts of LTA (Panel D) were significantly different between the $\Delta / \mathrm{ytT}$ and $\Delta d \mathrm{dltD}$ strains at $67 \mathrm{~h}$ (\#); while at $115 \mathrm{~h}, \Delta d l t A$ contained more LTA than the $\Delta l y t T$ and $\Delta l y t S$ strains $\left({ }^{\S} p<0.05\right.$; two-way ANOVA using developmental phase and $S$. mutans strains as factors, followed by Tukey's test). The plotted data are averages, and error bars indicate the SDs ( $n=6$ for single-species biofilms at 67 and $115 \mathrm{~h}$, and for mixed-species biofilms at $115 \mathrm{~h} ; n=10$ for mixed-species biofilms at $67 \mathrm{~h}$ ).

significant increase in soluble proteins was observed for $\Delta l y t T, \Delta d l t A$ and $\Delta d l t D$ strains from 67 to $115 \mathrm{~h}$ for single-species biofilms (Figure S2A). However, as mixed-species biofilms aged, no statistically significant changes were measured in protein content of the ECM (Figure S2B). The amount of soluble proteins in the ECM did not vary significantly at $67 \mathrm{~h}$ between the six strains evaluated in single-species biofilms. At $115 \mathrm{~h}$, the $\Delta g t f B$ strain contained the least amount of protein, compared to four of the other strains tested, except $\Delta l y t S$ (Figure S2A). Also, there were no differences in the amount of proteins recovered from the ECM of mixed-species biofilms at $67 \mathrm{~h}$, but at $115 \mathrm{~h}, \Delta g t f B$ biofilms were reduced in protein content, compared to UA159 and $\Delta d l t A$.
Correlation data between S. mutans population, biomass, protein, and matrix components (eDNA, LTA, WSP, ASP and proteins) for each S. mutans strain in single- and mixed-species biofilms

EPS, represented as WSP and ASP quantities, correlated positively and significantly in both single- and mixed-species biofilms of all strains as the biofilms aged $(r \geq 0.7974$; $p \leq 0.004$; Tables S1-S6). For the UA159 parental strain, the $S$. mutans population did not show significant correlation with other biofilm parameters in single-species biofilms, while in mixed-species biofilms strong positive correlations were found for biomass, biofilm proteins, LTA, WSP and ASP, meaning that as these biofilms aged and the $S$. mutans population increased, the quantity of these components also augmented (Table S1). Biomass 

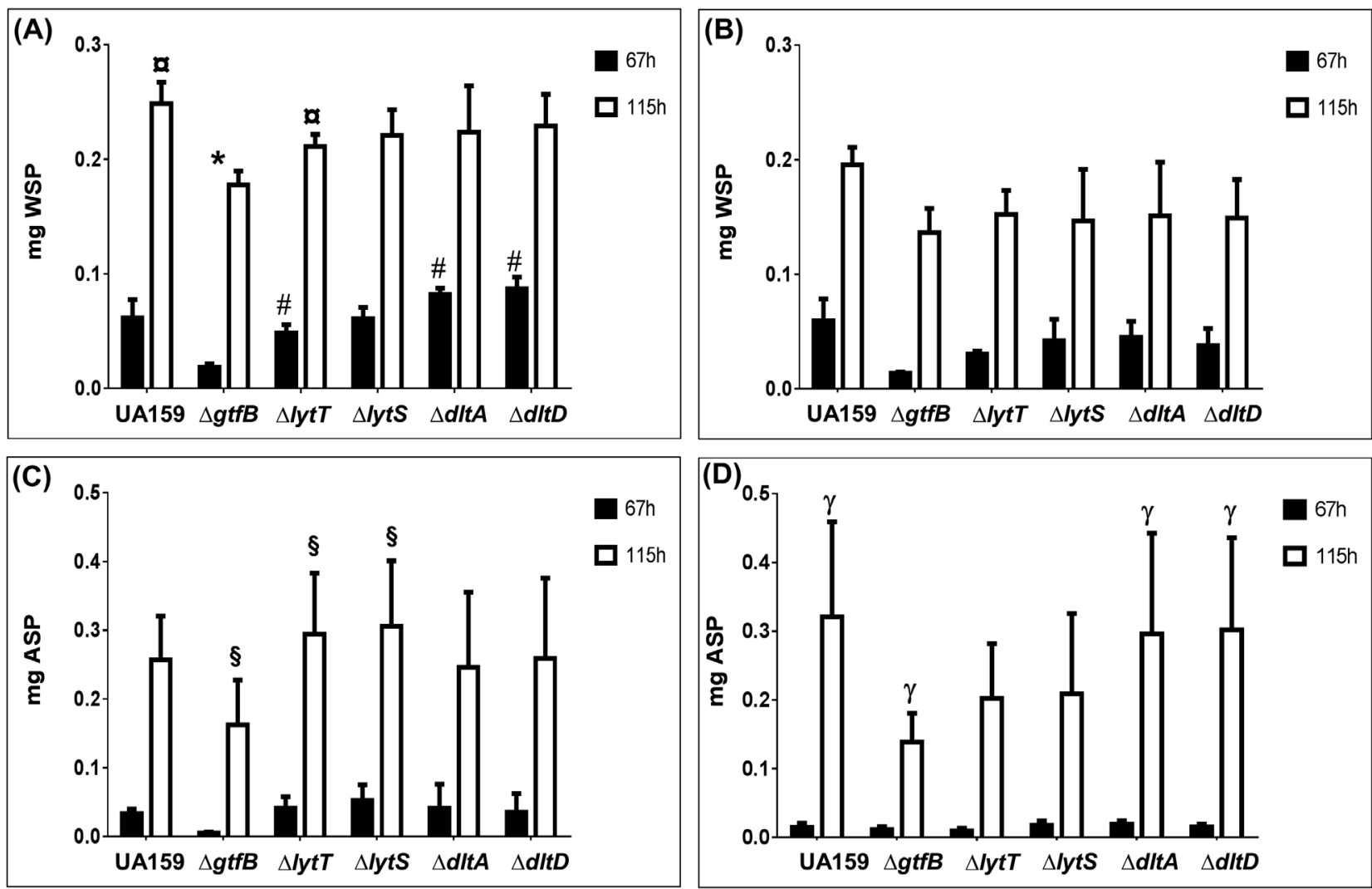

Figure 6. Water-soluble and -insoluble exopolysaccharides in the ECM of single- and mixed- species biofilms.

Notes: Total amounts of extracellular water-soluble exopolysaccharides (WSP) were measured from single- (Panel A) and mixed-species (Panel B) biofilms at $67 \mathrm{~h}$ (black bars) and $115 \mathrm{~h}$ (white bars). Total amounts of extracellular water-insoluble exopolysaccharides (ASP) were measured from single- (Panel C) and mixedspecies (Panel D) biofilms at $67 \mathrm{~h}$ (black bars) and $115 \mathrm{~h}$ (white bars). For both single- and mixed-species biofilms, there was a significant increase in the amount of WSP and ASP in the ECM between 67 to $115 \mathrm{~h}(p<0.05$; two-way ANOVA using developmental phase and S. mutans strains as factors, followed by Sidak's test symbols representing statistical analysis outcome are not depicted in the graphs). In single-species biofilms (Panel A), $\triangle g t f B$ contained less WSP in the ECM at both 67 and $115 \mathrm{~h}$, compared to all other strains tested, except $\Delta l y t T$, at $67 \mathrm{~h}\left({ }^{*} p \leq 0.03\right.$; two-way ANOVA using developmental phase and $S$. mutans strains as factors, followed by Tukey's test). The biofilm derived from the $\Delta l y t T$ strain was composed of less WSP at the $67 \mathrm{~h}$ time point (vs strains $\Delta d l t A$ and $\Delta d l t D ; \# p \leq 0.0278$ ), and at $115 \mathrm{~h}$ ( $v s$ parental strain; $x p=0.0099$; two-way ANOVA, followed by Tukey's test). In mixed-species biofilms (Panel B), there was no significant variation in WSP between strains at $67 \mathrm{~h}$ or at $115 \mathrm{~h}(p>0.05)$. ASP measurements were similar for all strains at $67 \mathrm{~h}$ in single-species biofilms (Panel C); while at $115 \mathrm{~h}$, the $\Delta g t f B$ strain contained less ASP (vs $\Delta l y t T$ and $\Delta l y t S$ strains; ${ }^{\circledR} p \leq 0.02$; two-way ANOVA using developmental phase and $S$. mutans strains as factors, followed by Tukey's test). In mixed-species biofilms, there were no differences between strains for ASP at $67 \mathrm{~h}$ (Panel D); however, at $115 \mathrm{~h}$, there was less ASP for the $\Delta g t f B$ strain ( $v s$ parental strain UA159, $\Delta d l t A$ and $\Delta d l t D ;{ }^{\vee} p \leq 0.02$; two-way ANOVA using developmental phase and S. mutans strains as factors, followed by Tukey's test). The plotted data are averages, and error bars indicate the SDs $(n=6$ for single-species biofilms at 67 and $115 \mathrm{~h}$, and for mixed-species biofilms at $115 \mathrm{~h} ; n=10$ for mixed-species biofilms at $67 \mathrm{~h}$ ).

Table 2. Glycosyl linkage data of the water-soluble and -insoluble exopolysaccharides from the ECM of mixed-species biofilms.

\begin{tabular}{|c|c|c|c|c|c|c|c|}
\hline \multirow[b]{2}{*}{ Biofilm } & \multicolumn{4}{|c|}{$\begin{array}{l}\text { Water-soluble exopolysaccha- } \\
\text { rides }\end{array}$} & \multicolumn{3}{|c|}{$\begin{array}{c}\text { Water-insoluble exopol- } \\
\text { ysaccharides }\end{array}$} \\
\hline & $\begin{array}{c}\% \\
\text { T-Glc }\end{array}$ & $\begin{array}{c}\% \\
3-G l c\end{array}$ & $\begin{array}{c}\% \\
4-G l c\end{array}$ & $\% 3,4-$ & $\begin{array}{c}\% \\
3-G l c\end{array}$ & $\begin{array}{c}\% \\
4-G l c\end{array}$ & $\begin{array}{l}\% 3,4- \\
\text { Glc }\end{array}$ \\
\hline UA159 & $\begin{array}{l}26.7 \pm \\
1.0\end{array}$ & & & & & & $\begin{array}{l}7.2 \pm \\
4.0\end{array}$ \\
\hline IgtfB & & & & & $\begin{array}{l}34.5 \pm \\
4.8\end{array}$ & $\begin{array}{l}56.6 \pm \\
2.1\end{array}$ & $\begin{array}{l}8.8 \pm \\
2.7\end{array}$ \\
\hline$\Delta l y t T$ & $\begin{array}{l}34.7 \pm \\
0.2\end{array}$ & $\begin{array}{l}43.3 \pm \\
0.3\end{array}$ & $\begin{array}{l}20.6 \pm \\
0.5\end{array}$ & $\begin{array}{l}1.4 \pm \\
0.0\end{array}$ & $\begin{array}{l}36.7 \pm \\
4.5\end{array}$ & $\begin{array}{l}63.3 \pm \\
4.5\end{array}$ & $\begin{array}{l}0.0 \pm \\
0.0\end{array}$ \\
\hline$\Delta l y t S$ & $\begin{array}{l}30.0 \pm \\
1.0\end{array}$ & $\begin{array}{l}45.8 \pm \\
0.5\end{array}$ & $\begin{array}{l}21.8 \pm \\
1.6\end{array}$ & $\begin{array}{l}2.4 \pm \\
0.1\end{array}$ & $\begin{array}{l}31.7 \pm \\
26.5\end{array}$ & $\begin{array}{l}68.3 \pm \\
26.5\end{array}$ & $\begin{array}{l}0.0 \pm \\
0.0\end{array}$ \\
\hline$\Delta d l t A$ & $\begin{array}{l}35.1 \pm \\
0.5\end{array}$ & $\begin{array}{l}39.6 \pm \\
0.5\end{array}$ & $\begin{array}{l}23.6 \pm \\
0.3\end{array}$ & $\begin{array}{l}1.7 \pm \\
0.2\end{array}$ & $\begin{array}{l}0.0 \pm \\
0.0\end{array}$ & $\begin{array}{l}100.0 \\
\pm 0.0\end{array}$ & $\begin{array}{l}0.0 \pm \\
0.0\end{array}$ \\
\hline$\Delta d l t D$ & $\begin{array}{l}42.1 \pm \\
2.2\end{array}$ & $\begin{array}{l}31.8 \pm \\
1.8\end{array}$ & $\begin{array}{l}22.9 \pm \\
0.9\end{array}$ & $\begin{array}{l}3.3 \pm \\
0.5\end{array}$ & $\begin{array}{l}21.3 \pm \\
24.8\end{array}$ & $\begin{array}{l}65.7 \pm \\
43.2\end{array}$ & $\begin{array}{l}13.0 \pm \\
18.4\end{array}$ \\
\hline
\end{tabular}

Note: Data shown are average \pm SD of the percentage for each type of linkage. amounts correlated positively and significantly with LTA, WSP and ASP while there was a negative correlation with eDNA in both single and mixed species biofilm cultures containing UA159. Moreover, eDNA correlated negatively with all other ECM components, being statistically significant for LTA, WSP and ASP, but not for proteins. In addition, LTA correlated positively with WSP, ASP and proteins in the ECM, and correlation was statistically significant only for WSP in both biofilm types of UA159. However, the deletion of specific genes influenced the correlation between biofilm components.

For the $\Delta g t f B$ strain, the $S$. mutans population showed significant negative correlation with eDNA, WSP and ASP in single-species biofilms; however, in mixed-species biofilms there was a positive correlation between $S$. mutans 
population and EPS (WSP and ASP) and biomass (Table S2). Biomass correlated positively with eDNA, LTA, WSP and ASP, being statistically significant for eDNA and WSP in single-species $\Delta g t f B$; but biomass correlated positively only with WSP and ASP in mixed-species $\Delta g t f B$. For the $\Delta g t f B$ strain, eDNA amounts correlated strongly and positively with LTA, WSP and ASP in single-species, while there were no significant correlations of eDNA with any component in mixed-species biofilms. For LTA, there were no strong correlations with biofilms components in single- or mixed-species biofilms by $\triangle g t f B$ (except eDNA in single-species).

The $S$. mutans population of the $\Delta l y t T$ strain showed no significant correlation with any of the biofilm components in single-species biofilms, but it had significant strong positive correlations with biomass, eDNA, LTA, WSP and ASP in mixed-species biofilms (Table S3). In biofilms with $\Delta l y t T$ strain, the biomass correlated positively with WSP, ASP and proteins in the ECM in single-species biofilms ( $r=-0.748 ; p=0.0051)$; while biomass only correlated positively and significantly with LTA and WSP in mixed-species biofilms. Interestingly, eDNA correlation with biofilm components was different in single- $v s$ mixed-species biofilms of $\Delta l y t T$ strain. In single-species biofilms of $\Delta l y t T$, eDNA showed strong and negative significant correlation with EPS (WSP and ASP) and proteins in the ECM; while in mixed-species biofilms the strong correlations of eDNA with WSP and ASP were positive. LTA showed significant positive correlation only with WSP in the ECM $(r=0.6893 ; p=0.0190)$.

The $S$. mutans population of $\Delta l y t S$ strain showed significant negative correlation with biomass, LTA and WSP in single-species biofilms, while in mixed-species biofilms it correlated positively with biomass, eDNA, WSP and ASP (Table S4). Biomass of $\Delta l y t S$ biofilms showed significant positive correlation with WSP and ASP in a single-species setting, but not correlated with eDNA and ASP in the mixed-species model. eDNA content in the ECM of $\Delta l y t S$ strain biofilms showed a significant strong positive correlation with ASP in single-species setting, and with WSP and ASP in the mixed-species model as biofilms aged. In addition, LTA did not show strong correlations with components of both single- and mixed-species biofilms formed by the $\Delta l y t S$ strain.

For the $\Delta d l t A$ strain, the $S$. mutans population only showed significant positive correlation with WSP and ASP in mixed-species biofilms (Table S5). Biomass yielded a significant positive correlation with WSP and ASP in both biofilm types, and also with proteins only in a single-species setting of $\Delta d l t A$ strain. eDNA did not correlate with any parameters evaluated in both biofilm types of $\Delta d l t A$, except with proteins in mixed-species biofilms. LTA only showed significant positive correlation with ASP and proteins in the ECM of $\triangle d l t A$ mixed-species biofilms.
The S. mutans population of $\Delta d l t D$ strain showed no significant correlation with any of the biofilm components in single-species biofilms, while in mixed-species biofilms it correlated positively with biomass, proteins in biofilm, WSP and ASP (Table S6). The biomass of $\Delta d l t D$ biofilms showed significant positive correlation with proteins in biofilm, WSP and ASP in both biofilm types, while it also correlated with LTA and proteins in the ECM in single-species setting, and eDNA only presented significant negative correlation with WSP in mixed-biofilms of $\Delta d l t D$. In addition, LTA showed a significant positive correlation with ASP in both biofilm types of $\Delta d l t D$, and with WSP in a single-species biofilm.

\section{3-D architecture, quantity of bacteria, and EPS content in the single- and mixed-species biofilms}

The 3-D architectural organization was evaluated in $115 \mathrm{~h}$-old single- and mixed-species biofilms, and representative images are depicted in Figure 7. As expected, single-species biofilms formed by the parental strain, UA159, presented well-defined bacterial microcolonies enmeshed with an ESP matrix, while biofilms created by the $\triangle g t f B$ strain lacked defined microcolonies (Koo et al. 2010; Xiao et al. 2012). While the $\Delta g t f B$ strain does not have a functional GtfB enzyme for ASP production on bacterial surfaces, the intact GtfC enzyme has affinity for the sHA surface, forming a carpet-like EPS matrix on it (Koo et al. 2010; Xiao et al. 2012). The 3-D structures of single-species biofilms of the $\Delta l y t T, \Delta l y t S$ and $\Delta d l t A$ strains were similar, and displayed larger, more defined microcolonies, compared to UA159, though biofilms of $\Delta d l t D$ contained areas devoid of microcolonies. Therefore, inactivation of $d l t D$ appeared to have a greater influence on the 3-D assembly of $S$. mutans single-species biofilms (Figure 7, top panels).

The 3-D structure of mixed-species biofilms was strikingly different from that of single-species biofilms, which was also evident in measurements of the biovolume (biomass), EPS content (Figure 8), and the profile of the distribution of bacteria and EPS (Figures 9 and 10). In the mixed-species biofilms, well-defined microcolonies were essentially absent in all biofilms containing the $S$. mutans mutant strains, and sparsely observed in biofilms with the S. mutans parental strain, UA159.

\section{Discussion}

All biological events occurring in the construction of a biofilm are germane, especially those involved in ECM formation, to the development of effective approaches for the prevention and treatment of pathogenic biofilms. Thus, the present study evaluated the biochemical composition 

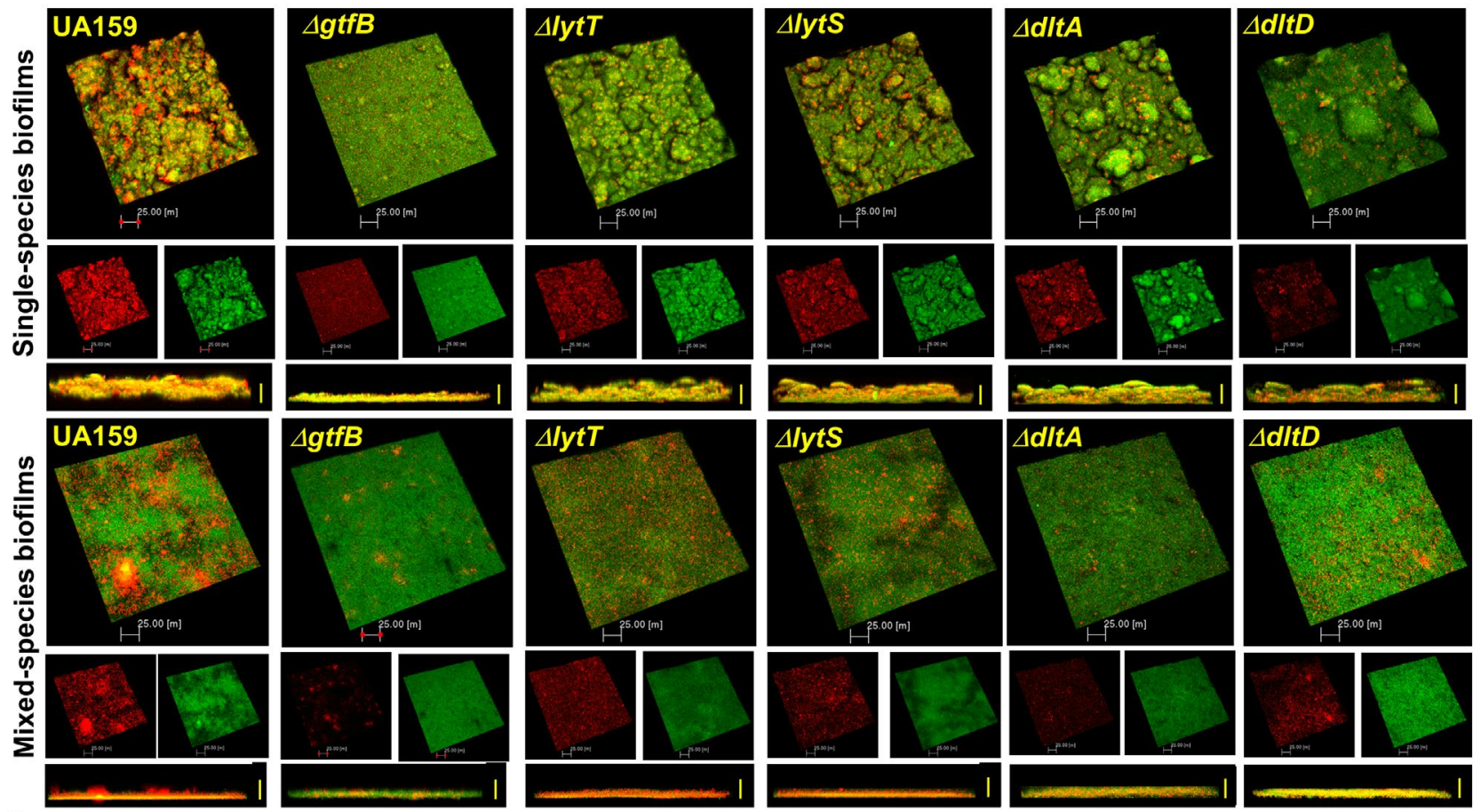

Figure 7.3D architecture of the single- and mixed species biofilms.

Notes: Representative 3-D renderings of single- (upper panel) and mixed-species (lower panel) biofilms formed by S. mutans parental (UA159) and mutant strains $(\Delta g t f B, \Delta l y t T, \Delta l y t S, \Delta d l t A$, and $\Delta d l t D)$ at $115 \mathrm{~h}$. Biofilms were labeled as described in Materials and methods. Nucleic acids were stained green with SYTO 9 green fluorescent nucleic acid stain and the extracellular polysaccharides are stained red with Alexa Fluor 647 -labeled dextran conjugate. The imaging was performed using a Zeiss LSM 780 microscope equipped with a 20x objective lens. The larger image in each set represents the overlaid images of the red and green channels. The lower image is a cross-section of the biofilm with overlaid images. Scale bar: $25 \mu \mathrm{m}$.
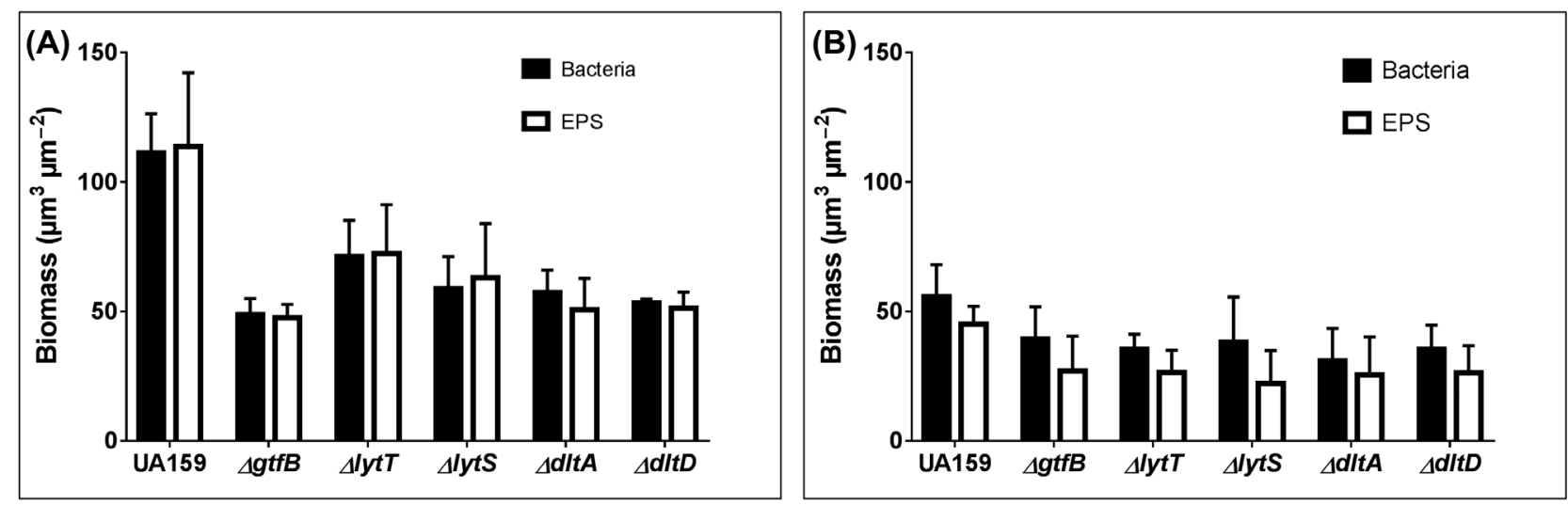

Figure 8. Biovolume of bacteria and EPS in single- and mixed species biofilms.

Notes: Biovolume is represented as biomass $\left(\mu \mathrm{m}^{3} \mu \mathrm{m}^{-2}\right.$ ) of bacteria (black bars) and EPS (white bars) for single- (Panel A) and mixed-species (Panel B) biofilms at $115 \mathrm{~h}$. (Panel A) For single-species biofilms, two-way ANOVA demonstrated no interaction between distinct strains and biofilm component (bacteria vs EPS). Sidak's multiple comparison test demonstrated significant differences for all mutants compared to parental strains $(p<0.05)$, except for $\Delta / y t T$. Moreover, no differences were found by comparing all mutants against each other for both bacteria and EPS biomass. The plotted data are averages, and error bars indicate the SDs $(n=4)$. (Panel B) For mixed-species biofilms, two-way ANOVA demonstrated no interaction between distinct strains and biofilm component, but distinct strains factor and biofilm component factor (bacteria vs EPS) were significant $(p<0.0001)$. The Tukey's multiple comparison test demonstrated that bacteria and EPS biomass of biofilm with parental strain UA159 was significantly different (higher) than all deletion strains tested $(p<0.05)$. The plotted data are averages, and error bars indicate the SDs ( $n=4$ for single-species biofilms; $n=8$ for mixed-species biofilms).

of the ECM, detecting the presence and content of eDNA, LTA, water-soluble and -insoluble exopolysaccharides, and soluble proteins at various stages of biofilm development, as eDNA interacts with EPS in the early phases of biofilm development, while LTA participates in the maturation of matrix and biofilm. In general, as expected, the components were more abundant in $115 \mathrm{~h}$-old biofilms, although there was a relatively greater amount of these components in single-species biofilms, compared to mixed-species biofilms. This phenomenon can be explained by the presence of other non-mutans species in mixed-species biofilms, that may interfere with, or 

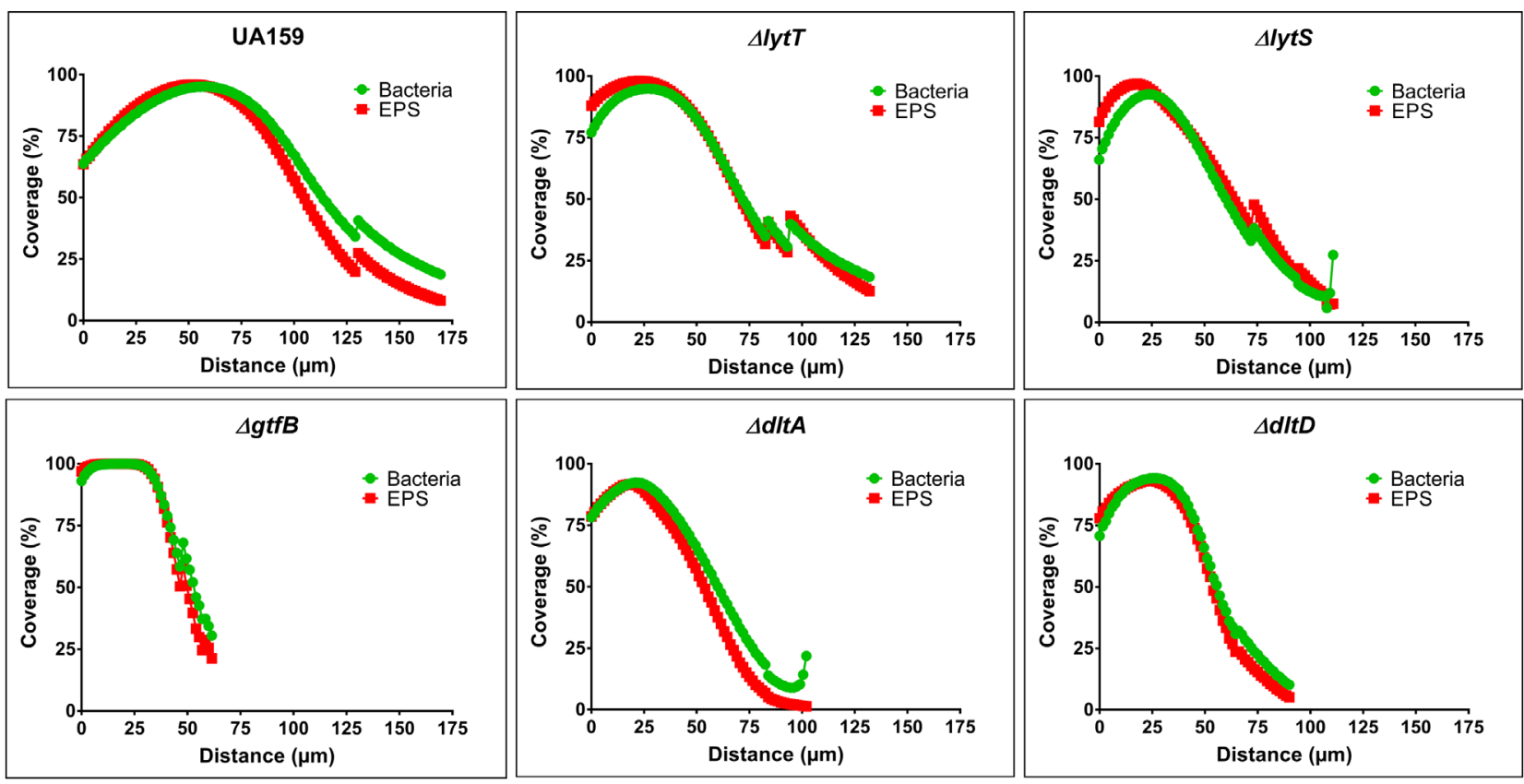

Figure 9. Profile of the distribution of bacteria and EPS in each of the single-species biofilms.

Note: The data shown are the mean percentage coverage per area from the interface substratum/biofilm (HA disk) to the top (outer layer) of each biofilm at $115 \mathrm{~h}$ $(n=12$ images per biofilm).
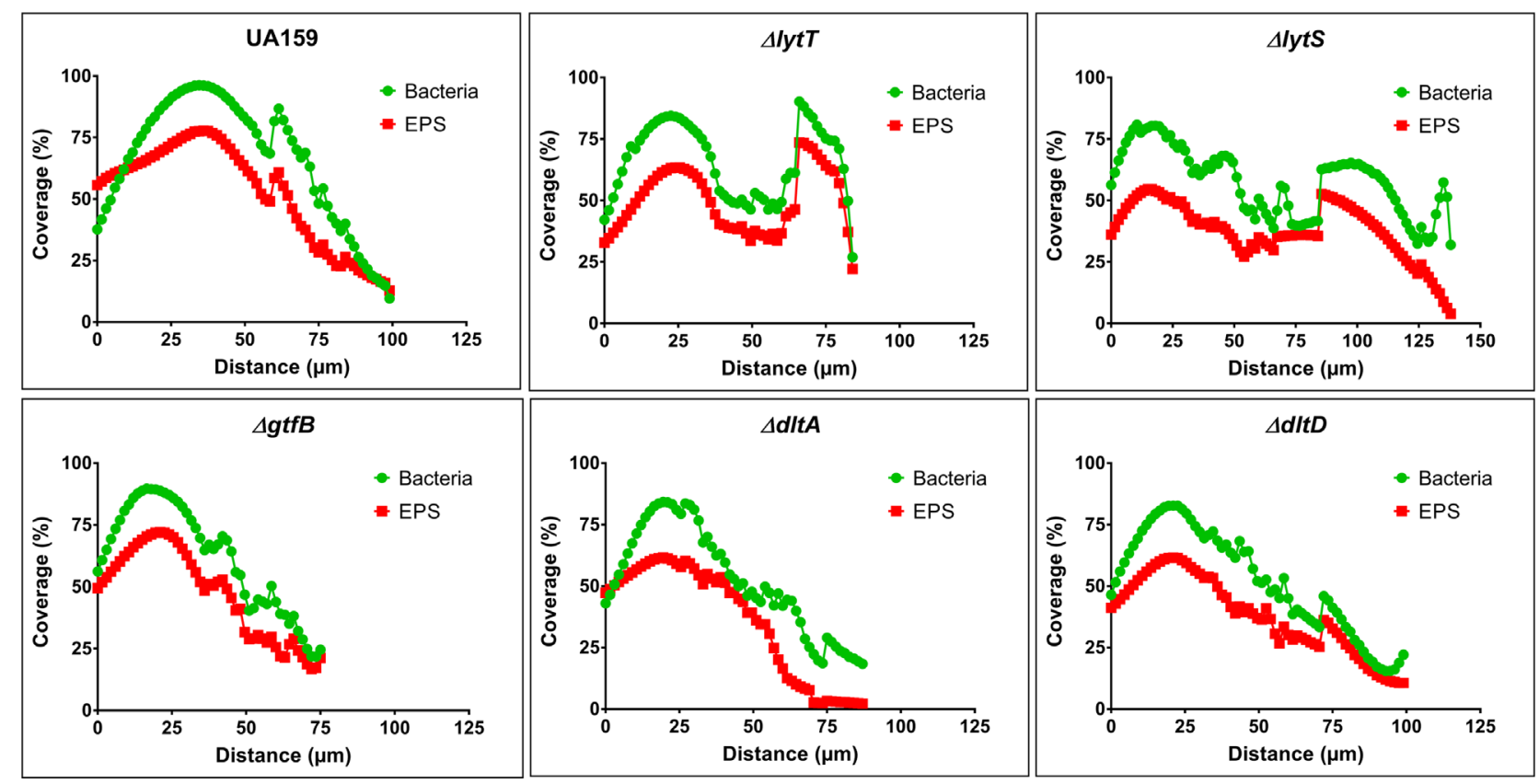

Figure 10. Profile of the distribution of bacteria and EPS in each of the mixed-species biofilms.

Note: The data shown are the mean percentage coverage per area from the interface substratum/biofilm (HA disk) to the top (outer layer) of each biofilm at $115 \mathrm{~h}$ ( $n=15$ images per biofilm).

stimulate, specific components in the ECM of $S$. mutans. Furthermore, the deletion of specific $S$. mutans genes not only affected ECM composition, but also the population dynamics and 3-D architecture of biofilms.

The relationship between the abundance of carbohydrates (sucrose and starch), and incubation time, could help increase adhesion of microorganisms to the HA surface and build-up of matrix. In the modern diet, sucrose and starch are ingested simultaneously or interleaved, and starch can increase the cariogenic power of sucrose (Ribeiro et al. 2005; Duarte et al. 2008; Klein et al. 2009, 2010). Moreover, the presence of both sucrose and starch 
in biofilm cultures and the presence of other species (in a mixed-species setting) have been shown to enhance the expression of gene products in S. mutans (including eDNA and LTA) that are involved with ECM assembly in cariogenic biofilms (Klein et al. 2010, 2012).

In mixed-species biofilms, S. mutans and S. gordonii were present throughout the developmental process, while A. naeslundii was not observed at 115 h. S. mutans has been shown to be the cariogenic species that increases in population over time, under appropriate conditions, but is not always the most abundant organism in the early colonizer community on the tooth surface (Takahashi and Nyvad 2011; Mattos-Graner et al. 2014). Thus, favorable conditions, such as an abundance of fermentable carbohydrates to produce acids and to serve as a substrate (sucrose) for enzymes that synthesize soluble and insoluble EPS, can promote the growth of $S$. mutans (Bowen and Koo 2011).

S. gordonii and A. naeslundii can produce alkali using salivary substrates such as arginine, via the arginine deiminase system (S. gordonii ADS) and urea, via the urease enzyme (A. naeslundii) (Liu et al. 2012; Nascimento et al. 2013). Presumably, alkali-producing bacteria protect against the acidification of the biofilm and prevent the growth or dominance of cariogenic microorganisms that thrive under acidic conditions, while also helping to prevent the deleterious effects of demineralization. However, in the current model, the presence of both S. gordonii and A. naeslundii did not hinder acidification and predominance by the $S$. mutans population at later stages of biofilm development. Alkali production by $A$. naeslundii and $S$. gordonii may not overcome the acid produced by $S$. mutans during biofilm growth. Of note, $S$. gordonii was the most prevalent species in the early stages $(29-53 \mathrm{~h})$ of biofilm formation, consistent with its role as an early colonizer. This observation could be explained by the production of hydrogen peroxide by the organism, thereby establishing a niche during the adhesion process (Kreth et al. 2008); or, because this species has more affinity to the salivary pellicle (Nobbs et al. 2009). Thus, those two species did not outcompete $S$. mutans in the present model, and may have benefited from the EPS matrix assembled by $S$. mutans, even in the biofilms formed by deletion strains.

The increased arginine metabolism associated with a dentifrice containing arginine may exert an inhibitory effect on caries (Nascimento et al. 2014). However, species that encode ADS and urease are also capable of acidogenesis and aciduricity. But, whether the use of arginine and urea would serve to neutralize the acidic environment in biofilms, or whether these systems would enable competition against other aciduric species, affecting the construction of the ECM, needs to be further elucidated. A recent study used topical application of arginine in a biofilm model similar to the one used in this study (however, without saliva in the culture medium or alternating carbohydrate concentrations). The results of that study demonstrated that high concentrations of arginine have a negative influence on the amount of EPS in the matrix of mixed-species biofilms (He et al. 2016). Therefore, understanding population dynamics is important for the application of strategies to control pathogenic biofilm formation (ie inactivation of specific species or the metabolic pathways of such species).

Clearly, all components of the ECM evaluated were present in the biofilms. The three species used in the mixed-species model could be producing eDNA and LTA and protein (Neuhaus and Baddiley 2003; Kreth et al. 2009), although S. mutans appears to be the main pathogen under the cariogenic conditions mimicked here. In the transition phase between $67 \mathrm{~h}$ and $115 \mathrm{~h}$ (mature biofilm), both eDNA and LTA are released in the ECM while soluble and insoluble EPS are being synthetized, resulting in an increased amount of these components in the mature biofilm in both single- and mixed-species biofilms. The gtfB gene product has been shown to influence the formation of cariogenic biofilms and the composition of the matrix components (Xiao et al. 2012). Fewer ASP measured in biofilms formed by the $\Delta g t f B$ strain was anticipated, as the enzyme encoded by this gene ( $\mathrm{GtfB})$ is one of the glucosyltransferases responsible for the synthesis of ASP (Bowen and Koo 2011; Xiao et al. 2012).

The initial hypothesis was that eDNA release could be due to expression of lytST (Klein et al. 2010); thus, using strains deleted for these genes should negatively affect the presence of eDNA in the ECM. However, the mutant strains $\Delta l y t T$ and $\Delta l y t S$ generally had the highest amounts of eDNA, when compared to all strains tested in singleand mixed- species biofilms, reinforcing that both lyt $T$ and $l y t S$ genes participate in eDNA modulation of the extracellular matrix, as previously demonstrated (Ahn et al. 2010; Liao et al. 2014). However, it was expected that the amount of eDNA would be lower when compared to the S. mutans parental strain. Therefore, it is postulated that the discrepancy observed may be because the lack of a functional lytST TCS could increase cell wall turnover, thereby increasing the secretion of microvesicles containing eDNA, or trigger additional pathways that culminate in augmented release of DNA to the matrix. Thus, the lack of $\Delta l y t T$ or $\Delta l y t S$ could help the formation of a highly cohesive matrix that would increase the production of exopolysaccharides and eDNA, which provides a negative charge to the ECM. The encounter of a negatively charged net of extracellular polymeric substances by a positively charged agent (that should penetrate or diffuse into it) can reduce the concentration and speed of this agent to reach microbial cells hindering its effects on the 
cells (Flemming and Wingender 2010). Thus, the higher amounts of eDNA detected in biofilms containing $\Delta l y t T$ or $\Delta l y t S$ could influence the action of preventive agents or cationic therapeutics (eg chlorhexidine; Xiao et al. 2012).

Clearly, other factors must be influencing the amount of eDNA. It is possible that the presence of $l y t T$ or $l y t S$ in the $\Delta l y t S$ and $\Delta l y t T$ strain, respectively, enables $S$. mutans to effectively release eDNA. Additional studies using a lytST double mutant may elucidate the exact extent of this system in eDNA release. The absence of lyt $T$ did result in an increase in soluble EPS content from 67 to $115 \mathrm{~h}$ in single-species biofilms. However, when both $\Delta l y t T$ and $\Delta l y t S$ were first grown as single-species biofilms on sHA disks and evaluated (Chatfield et al. 2005), it was found that there were no marked differences from the parental strain biofilm for biomass, and soluble and insoluble EPS after five days of growth. Previously, both $\triangle d l t A$ and $\Delta d l t D$ were shown to have poor acid survival in planktonic cultures and single-species biofilm formation deficiencies on polystyrene surface in the presence of sucrose or glucose (Quivey et al. 2015), which were not observed here. The differences could be explained as the result of distinct parameters, including cycling low concentrations of sucrose and a combination of sucrose and starch instead of high sucrose only, the presence of $25 \%$ saliva in the culture medium and the biofilm substratum. Consequently, perhaps $l y t T$ would be a candidate for further research. In some bacterial species, such as Enterococcus faecalis and Pseudomonas aeruginosa, part of the biofilm population sacrifices itself for the community, via cell lysis, and releases eDNA into the biofilm milieu (Thomas et al. 2009; Turnbull et al. 2016). However, it is currently unknown whether $S$. mutans may behave similarly.

The negative correlation of eDNA amount with biomass and other ECM components as UA159 single- and mixed-species biofilms aged points to eDNA having a role in the early stages of ECM and biofilm development, as suggested previously (Liao et al. 2014). The role of eDNA in the early stages is corroborated by the data found for the $\Delta g t f B$ strain in single-species biofilms where less EPS (both ASP and WSP) was detected and EPS correlated positively with the amount of eDNA, because ECM accumulation is slower over time, maintaining $\Delta g t f B$ biofilms in a 'younger' state compared to the biofilms of the parental strain that present a massive build-up as they aged. The absence of lytS (sensor kinase) and lyt $T$ (response regulator) augmented the amount of eDNA in the ECM. Furthermore, eDNA presented a strong positive correlation with EPS as biofilms aged for single- (ASP) and mixed-species (WSP and ASP) for $\Delta l y t S$, while in $\Delta l y t T$ biofilms, eDNA correlated negatively with EPS in a single-species setting, but positively in a mixed-species model. There is no clear explanation for the contradictory behavior of eDNA and EPS in the matrix of both the $\Delta l y t T$ and $\Delta l y t S$ strains. Possible explanations are (1) that there is an overlap of controlling mechanisms to augment eDNA in the ECM at later stages of biofilm development for $\Delta l y t S$ and (2) that in a mixed-species model, other species contribute to the amount of eDNA in the ECM.

The gene products of the operon dltABCD are associated with LTA metabolism (Neuhaus and Baddiley 2003). The increase in extracellular LTA in the ECM of biofilms formed by $\Delta d l t A$ and $\Delta d l t D$ was unexpected, thus, the absence of either dltA or dltD in the dltABCD operon may trigger an unknown compensatory pathway, causing an increase in LTA release to the matrix by these strains. Therefore, it is postulated that there could be an increase in the remodeling of the cell wall, making these genes relevant as targets in future anti-biofilm therapies, as previously suggested (Qiu et al. 2015). Moreover, the D-alanization process mediated by a functional dlt operon has been shown to confer resistance to cationic antimicrobial agents in Streptococcus pneumoniae (Kovács et al. 2006), but how it would affect strategies against cariogenic biofilms warrants further studies. Moreover, it was demonstrated here that the loss of $d l t A$ and $d l t D$ influenced the production of all components of the extracellular matrix, particularly an elevated ASP content in single-species biofilms (at $67 \mathrm{~h}$ ) and increased protein content in the ECM (at $115 \mathrm{~h}$ ). High levels of LTA have been measured in in vivo plaque biofilms induced by sucrose (Rölla et al. 1980), and the presence of LTA in the ECM was confirmed in the experiments described here, showing an association with increased amounts of EPS.

As EPS are a virulence determinant in cariogenic biofilms (Koo et al. 2013), LTA metabolism should be taken into account to prevent the development of these biofilms, especially since LTA was positively correlated with WSP, ASP and proteins in the ECM, indicating that it may play a role in all stages of biofilm development, different from eDNA for UA159. The lack of strong and significant correlations between LTA and other components (except with eDNA in single-species biofilms) in $\triangle g t f B$ biofilms reinforces the theory that LTA is needed for ECM assembly and bulky accumulation over time. In addition, eDNA and LTA showed significantly strong negative correlations in UA159 single- and mixed-species biofilms and positive correlations for $\Delta g t f B$ in single-species biofilms. Furthermore, eDNA and LTA did not show significant correlation in the $\Delta l y t S, \Delta l y t T, \Delta d l t A$ and $\Delta d l t D$ strains, although in mixed-species biofilms of $\Delta l y t S$ and $\Delta l y t T$ there was moderate positive correlation as the biofilm aged because the other two species also contributed to the eDNA in the ECM.

Furthermore, the 3-D architecture, the EPS and bacterial biovolume of single- and mixed-species biofilms 
were strikingly distinct. In single-species biofilms, well-defined microcolonies were detected for all strains, except for $\Delta g t f B$. The presence of both $S$. gordonii and A. naeslundii negatively influenced (1) the assembly of well-defined microcolonies in all biofilms (although the UA159 parental strain had some sparsely distributed), and (2) biofilm accumulation, leading to lower EPS and bacterial biovolume. Both factors can influence the diffusion of metabolites and how antimicrobial agents reach the microorganisms. In previous studies, it was demonstrated that inside large microcolonies, acidic niches can be created that could promote tooth demineralization (Xiao et al. 2012; Hwang et al. 2016). The lack of distinct microcolonies could be due to the influence of $S$. gordonii and $A$. naeslundii on the construction and assembly of the 3-D structure of microcolonies, such that the EPS is spread across the biofilm and not concentrated at specific areas, appearing as a carpet-like morphology rather than the 'peaks and valleys' observed in single-species biofilms. It is hypothesized that this may be occurring because of localized alkali production by S. gordonii and A. naeslundii that can interfere with the activity of Gtfs and glucan binding proteins (Gbps), possibly hindering the synthesis of glucans and/or the cross-linking of the glucans being produced, thereby affecting the 3 -D structure.

No in vitro model can mimic perfectly the diversity of the microorganisms that the mouth harbors, and none of them contain or reproduce all the factors that drive the composition of the oral microbiota and biofilm architecture. Nevertheless, it is critical to use mixed-species biofilms to evaluate the extent of potential virulence traits of a pathogen naturally living in a polymicrobial environment (such as the oral cavity). This is supported by the data from this study demonstrating that single- and mixed-species biofilms were not significantly different in terms of their biochemical composition/quantification of ECM components; however, the 3-D structure, and its quantitative features, showed a distinct scenario that must be considered when interpreting research data.

In summary, the current work provides evidence that both eDNA and LTA are present concomitantly with soluble and insoluble EPS in the biofilm matrix, contributing to the 3-D structure. Specifically, eDNA and LTA interact with EPS in the extracellular matrix of cariogenic $S$. mutans biofilms at distinct developmental phases, with eDNA being an important factor in the early stages and LTA in the later stages. Therefore, preventative and/or therapeutic interventions affecting the development of biofilms, specifically ECM components, could minimize the pathogenicity of virulent biofilms. Future studies should determine the best genetic targets to prevent the accumulation of those biofilms and to reduce the burden of dental caries.

\section{Acknowledgements}

The present research is part of the Master's dissertation by M.C.C.P.

\section{Disclosure statement}

No potential conflict of interest was reported by the authors.

\section{Funding}

The authors' research was supported in part by a research grant from the São Paulo Research Foundation (FAPESP [grant number 2014/05423-0] to M.I.K.) and scholarship ([grant number 2014/21355-4] to M.C.C.P.). T.F.N. received a scholarship from the National Counsel of Technological and Scientific Development (CNPq, PIBIC [grant numbers 29716, 35803, 39550, and 41910]). Additional funding was provided by the National Institute for Dental and Craniofacial Research [grant number DE017425-06] (R.G.Q Jr). The authors are thankful to Dr Sebastião Pratavieira (Confocal Core Facility, São Carlos Institute of Physics - University of Sao Paulo) for technical assistance during confocal fluorescence imaging of the biofilms. The confocal microscope facility used in this study was financed by FAPESP [grant number 2009/54035-4].

\section{ORCID}

Robert G. Quivey (D) http://orcid.org/0000-0001-8204-838X Marlise I. Klein iD http://orcid.org/0000-0002-7916-1557

\section{References}

Ahn SJ, Rice KC, Oleas J, Bayles KW, Burne RA. 2010. The Streptococcus mutans Cid and Lrg systems modulate virulence traits in response to multiple environmental signals. Microbiology. 156:3136-3147. doi:10.1099/ mic.0.039586-0.

Ajdić D, McShan WM, McLaughlin RE, Savić G, Chang J, Carson MB, Primeaux C, Tian R, Kenton S, Jia H, et al. 2002. Genome sequence of Streptococcus mutans UA159, a cariogenic dental pathogen. Proc Natl Acad Sci U S A. 99:14434-14439.

Bergeron LJ, Morou-Bermudez E, Burne RA. 2000. Characterization of the fructosyltransferase gene of Actinomyces naeslundii WVU45. J Bacteriol. 182:3649-3654. doi:10.1128/JB.182.13.3649-3654.2000.

Bowen WH. 2016. Dental caries - not just holes in teeth! A perspective. Mol Oral Microbiol. 31:228-233. doi:10.1111/ omi.2016.31.issue-3.

Bowen WH, Koo H. 2011. Biology of Streptococcus mutans derived glucosyltransferases: role in extracellular matrix formation of cariogenic biofilms. Caries Res. 45:69-86. doi:10.1159/000324598.

Bradford MM. 1976. A rapid and sensitive method for the quantitation of microgram quantities of protein utilizing the principle of protein-dye binding. Anal Biochem. 72:248254. doi:10.1016/0003-2697(76)90527-3.

Branda SS, Vik S, Friedman L, Kolter R. 2005. Biofilms: the matrix revisited. Trends Microbiol. 13:20-26. doi:10.1016/j. tim.2004.11.006. 
Chatfield CH, Koo H, Quivey RG Jr. 2005. The putative autolysin regulator LytR in Streptococcus mutans plays a role in cell division and is growth-phase regulated. Microbiology. 151:625-631. doi:10.1099/mic.0.27604-0.

Chiu TH, Baker JJ. 1994. Lipoteichoic acid from Streptococcus sanguis is a natural glucosyl acceptor for glucosyltransferases. Biochem Biophys Res Commun. 202:1407-1412. doi:10.1006/bbrc.1994.2087.

Ciardi JE, Reilly JA, Haller RH, Bowen WH, Rølla G. 1981. The role of lipoteichoic acid in the adherence and colonization of oral streptococci. In Shockman GD, Wicken AJ, editors. Chemistry and biological activities of bacterial surface amphiphiles. New York (NY): Academic Press, Inc; p. 353364. doi:10.1099/mic.0.039586-0.

Ciardi JE, Rölla G, Bowen WH, Reilly JA. 1977. Adsorption of Streptococcus mutans lipoteichoic acid to hydroxyapatite. Scand J Dent Res. 85:387-391.

Das T, Sharma PK, Busscher HJ, van der Mei HC, Krom BP. 2010. Role of extracellular DNA in initial bacterial adhesion and surface aggregation. Appl Environ Microbiol. 76:34053408. doi:10.1128/AEM.03119-09.

Denapaite D, Brückner R, Hakenbeck R, Vollmer W. 2012. Biosynthesis of teichoic acids in Streptococcus pneumoniae and closely related species: lessons from genomes. Microb Drug Resist. 18:344-358. doi:10.1089/mdr.2012.0026.

Duarte S, Klein MI, Aires CP, Cury JA, Bowen WH, Koo H. 2008. Influences of starch and sucrose on Streptococcus mutans biofilms. Oral Microbiol Immunol. 23:206-212. doi:10.1111/j.1399-302X.2007.00412.x.

Dubois M, Gilles KA, Hamilton JK, Rebers PA, Smith F. 1956. Colorimetric method for determination of sugars and related substances. Anal Chem. 28:350-356. doi:10.1021/ ac60111a017.

Flemming HC, Wingender J. 2010. The biofilm matrix. Nat Rev Microbiol. 8:623-633.

Gross M, Cramton SE, Gotz F, Peschel A. 2001. Key role of teichoic acid net charge in Staphylococcus aureus colonization of artificial surfaces. Infect Immun. 69:34233426. doi:10.1128/IAI.69.5.3423-3426.2001.

Heydorn A, Nielsen AT, Hentzer M, Sternberg C, Givskov M, Ersbøll BK, Molin S. 2000. Quantification of biofilm structures by the novel computer program COMSTAT. Microbiology. 146:2395-2407. doi:10.1099/00221287-14610-2395.

Haisman RJ, Jenkinson HF. 1991. Mutants of Streptococcus gordonii Challis over-producing glucosyltransferase. J Gen Microbiol. 137:483-489. doi:10.1099/00221287-137-3-483.

He J, Hwang G, Liu Y, Gao L, Kilpatrick-Liverman L, Santarpia P, Zhou X, Koo H. 2016. L-arginine modifies the exopolysaccharide matrix and thwarts Streptococcus mutans outgrowth within mixed-species oral biofilms. J Bacteriol. 198:2651-2661. doi:10.1128/JB.00021-16.

Hwang G, Liu Y, Kim D, Sun V, Aviles-Reyes A, Kajfasz JK, Lemos JA, Koo H. 2016. Simultaneous spatiotemporal mapping of in situ $\mathrm{pH}$ and bacterial activity within an intact 3D microcolony structure. Sci Rep. 6:32841. doi:10.1038/ srep32841.

Jakubovics NS, Gill SR, Iobst SE, Vickerman MM, Kolenbrander PE. 2008. Regulation of gene expression in a mixed-genus community: stabilized arginine biosynthesis in Streptococcus gordonii by coaggregation with Actinomyces naeslundii. J Bacteriol. 190:3646-3657. doi:10.1128/JB.00088-08.
Kassebaum NJ, Bernabé E, Dahiya M, Bhandari B, Murray CJ, Marcenes W. 2015. Global burden of untreated caries: a systematic review and metaregression. J Dent Res. 94:650658. doi:10.1177/0022034515573272.

Klein MI, DeBaz L, Agidi S, Lee H, Xie G, Lin AH, Hamaker BR, Lemos JA, Koo H. 2010. Dynamics of Streptococcus mutans transcriptome in response to starch and sucrose during biofilm development. PLoS ONE. 5:e13478. doi:10.1371/ journal.pone.0013478.

Klein MI, Duarte S, Xiao J, Mitra S, Foster TH, Koo H. 2009. Structural and molecular basis of the role of starch and sucrose in Streptococcus mutans biofilms development. Appl Environ Microbiol. 75:837-841. doi:10.1128/AEM.01299-08.

Klein MI, Hwang G, Santos PH, Campanella OH, Koo H. 2015. Streptococcus mutans-derived extracellular matrix in cariogenic oral biofilms. Front Cell Infect Microbiol. 5:1-8.

Klein MI, Xiao J, Lu B, Delahunty CM, Yates JR III, Koo H. 2012. Streptococcus mutans protein synthesis during mixed-species biofilm development by high-throughput quantitative proteomics. PLoS ONE. 7:e45795. doi:10.1371/ journal.pone.0045795.

Koo H, Falsetta ML, Klein MI. 2013. The exopolysaccharide matrix: a virulence determinant of cariogenic biofilm. J Dent Res. 92:1065-1073. doi:10.1177/0022034513504218.

Koo H, Xiao J, Klein MI, Jeon JG. 2010. Exopolysaccharides produced by Streptococcus mutans glucosyltransferases modulate the establishment of microcolonies within multispecies biofilms. J Bacteriol. 192:3024-3032. doi:10.1128/JB.01649-09.

Kovács M, Halfmann A, Fedtke I, Heintz M, Peschel A, Vollmer W, Hakenbeck R, Bruckner R. 2006. A functional dlt operon, encoding proteins required for incorporation of $\mathrm{d}$-alanine in teichoic acids in gram-positive bacteria, confers resistance to cationic antimicrobial peptides in Streptococcus pneumoniae. J Bacteriol. 188:5797-5805. doi:10.1128/JB.00336-06.

Kreth J, Vu H, Zhang Y, Herzberg MC. 2009. Characterization of hydrogen peroxide-induced DNA release by Streptococcus sanguinis and Streptococcus gordonii. J Bacteriol. 191:62816291. doi:10.1128/JB.00906-09.

Kreth J, Zhang Y, Herzberg MC. 2008. Streptococcal antagonism in oral biofilms: Streptococcus sanguinis and Streptococcus gordonii interference with Streptococcus mutans. J Bacteriol. 190:4632-4640. doi:10.1128/JB.00276-08.

Kuramitsu HK, Wondrack L, McGuinness M. 1980. Interaction of Streptococcus mutans glucosyltransferases with teichoic acids. Infect Immun. 29:376-382.

Lemos JA, Abranches J, Koo H, Marquis RE, Burne RA. 2010. Protocols to study the physiology of oral biofilms. Methods Mol Biol. 666:87-102. doi:10.1007/978-1-60761-820-1.

Liao S, Klein MI, Heim KP, Fan Y, Bitoun JP, Ahn SJ, Burne RA, Koo H, Brady LJ, Wen ZT. 2014. Streptococcus mutans extracellular DNA is upregulated during growth in biofilms, actively released via membrane vesicles, and influenced by components of the protein secretion machinery. J Bacteriol. 196:2355-2366. doi:10.1128/JB.01493-14.

Liu YL, Nascimento M, Burne RA. 2012. Progress toward understanding the contribution of alkali generation in dental biofilms to inhibition of dental caries. Int J Oral Sci. 4:135-140. doi:10.1038/ijos.2012.54.

Marsh PD. 2003. Are dental diseases examples of ecological catastrophes? Microbiology. 149:279-294. doi:10.1099/ mic.0.26082-0. 
Mattos-Graner RO, Klein MI, Smith DJ. 2014. Lessons learned from clinical studies: roles of mutans streptococci in the pathogenesis of dental caries. Curr Oral Health Rep. 1:7078. doi:10.1007/s40496-013-0008-1.

Melsen B, Kaae O, Rölla G, Fejerskov O, Karring T. 1979. Penetration of ions in human dental plaque. Arch Oral Biol. 24:75-81. doi:10.1016/0003-9969(79)90178-X.

Melvaer KL, Helgeland K, Rölla G. 1972. Some physical and chemical properties of "soluble" and "insoluble" polysaccharides produced by strains of Streptococcus mutans and sanguis. Caries Res. 6:79.

Melvaer KL, Helgeland K, Rölla G. 1974. A charged component in purified polysaccharide preparations from Streptococcus mutans and Streptococcus sanguis. Arch Oral Biol. 19:589955. doi:10.1016/0003-9969(74)90077-6.

Nascimento MM, Browngardt C, Xiaohui X, Klepac-Ceraj V, Paster BJ, Burne RA. 2014. The effect of arginine on oral biofilm communities. Mol Oral Microbiol. 29:45-54. doi:10.1111/omi.12044.

Nascimento MM, Liu Y, Kalra R, Perry S, Adewumi A, Xu X, Primosch RE, Burne RA. 2013. Oral arginine metabolism may decrease the risk for dental caries in children. J Dent Res. 92:604-608. doi:10.1177/0022034513487907.

Neuhaus FC, Baddiley J. 2003. A continuum of anionic charge: structures and functions of D-alanyl-teichoic acids in grampositive bacteria. Microbiol Mol Biol Rev. 67:686-723. doi:10.1128/MMBR.67.4.686-723.2003.

Nobbs AH, Lamont RJ, Jenkinson HF. 2009. Streptococcus adherence and colonization. Microbiol Mol Biol Rev. 73:407-450. doi:10.1128/MMBR.00014-09.

Paes Leme AF, Koo H, Bellato CM, Bedi G, Cury JA. 2006. The role of sucrose in cariogenic dental biofilm formation - new insight. J Dent Res. 85:878-887. doi:10.1177/154405910608501002.

Perry JA, Cvitkovitch DG, Lévesque CM. 2009. Cell death in Streptococcus mutans biofilms: a link between CSP and extracellular DNA. FEMS Microbiol Lett. 299:261-266. doi:10.1111/fml.2009.299.issue-2.

Pratten J, Barnett P, Wilson M. 1998. Composition and susceptibility to chlorhexidine of multispecies biofilms of oral bacteria. Appl Environ Microbiol. 64:3515-3519.

Qiu W, Zheng X, Wei Y, Zhou X, Zhang K, Wang S, Cheng L, Li Y, Ren B, Xu X, et al. 2015. D-Alanine metabolism is essential for growth and biofilm formation of Streptococcus mutans. Mol Oral Microbiol. 31:435-444.

Quivey RG Jr, Grayhack EJ, Faustoferri RC, Hubbard CJ, Baldeck JD, Wolf AS, MacGilvray ME, Rosalen PL, ScottAnne K, Santiago B, et al. 2015. Functional profiling in Streptococcus mutans: construction and examination of a genomic collection of gene deletion mutants. Mol Oral Microbiol. 30:474-495. doi:10.1111/omi.2015.30.issue-6.

Reichmann NT, Gründling A. 2011. Location, synthesis and function of glycolipids and polyglycerolphosphate lipoteichoic acid in Gram-positive bacteria of the phylum Firmicutes. FEMS Microbiol Lett. 319:97-105. doi:10.1111/ fml.2011.319.issue-2.
Ribeiro CC, Tabchoury CP, Del Bel Cury AA, Tenuta LM, Rosalen PL, Cury JA. 2005. Effect of starch on the cariogenic potential of sucrose. Br J Nutr. 94:44-50. doi:10.1079/ BJN20051452.

Rice KC, Mann EE, Endres JL, Weiss EC, Cassat JE, Smeltzer MS, Bayles KW. 2007. The cidA murein hydrolase regulator contributes to DNA release and biofilm development in Staphylococcus aureus. Proc Nat Acad Sci. 104:8113-8118. doi:10.1073/pnas.0610226104.

Rogers JD, Palmer RJ Jr, Kolenbrander PE, Scannapieco FA. 2001. Role of Streptococcus gordonii amylase-binding protein A in adhesion to hydroxyapatite, starch metabolism, and biofilm formation. Infect Immun. 69:7046-7056. doi:10.1128/IAI.69.11.7046-7056.2001.

Rölla G, Oppermann RV, Bowen WH, Ciardi JE, Knox KW. 1980. High amounts of lipoteichoic acid in sucrose-induced plaque in vivo. Caries Res. 14:235-238. doi:10.1159/000260459.

Selwitz RH, Ismail AI, Pitts NB. 2007. Dental caries. Lancet. 369:51-59. doi:10.1016/S0140-6736(07)60031-2.

Spatafora GA, Sheets M, June R, Luyimbazi D, Howard K, Hulbert R, Barnard D, el Janne M, Hudson MC. 1999. Regulated expression of the Streptococcus mutans dlt genes correlates with intracellular polysaccharide accumulation. J Bacteriol. 181:2363-2372.

Sutcliffe IC, Hogg SD. 1993. Extraction of lipoteichoic acid from Streptococcus mutants with the non-ionic detergent Triton X-114. J Microbiol Methods. 17:215-225. doi:10.1016/01677012(93)90048-M.

Takahashi N, Nyvad B. 2011. The role of bacteria in the caries process: ecological perspectives. J Dent Res. 90:294-303. doi: $10.1177 / 0022034510379602$.

Thomas VC, Hiromasa Y, Harms N, Thurlow L, Tomich J, Hancock LE. 2009. A fratricidal mechanism is responsible for eDNA release and contributes to biofilm development of Enterococcus faecalis. Mol Microbiol. 72:1022-1036. doi:10.1111/mmi.2009.72.issue-4.

Turnbull L, Toyofuku M, Hynen AL, Kurosawa M, Pessi G, Petty NK, Osvath SR, Cárcamo-Oyarce G, Gloag ES, Shimoni $\mathrm{R}$, et al. 2016. Explosive cell lysis as a mechanism for the biogenesis of bacterial membrane vesicles and biofilms. Nat Commun. 7:11220. doi:10.1038/ncomms11220.

Vacca-Smith AM, Venkitaraman AR, Quivey RG Jr, Bowen WH. 1996. Interactions of streptococcal glucosyltransferases with $\alpha$-amylase and starch on the surface of salivacoated hydroxyapatite. Arch Oral Biol. 41:291-298. doi:10.1016/0003-9969(95)00129-8.

Xiao J, Klein MI, Delahunty CM, Yates III JR, Lu B, Heydorn A, Falsetta ML, Koo H. 2012. The exopolysaccharide matrix modulates the interaction between $3 \mathrm{D}$ architecture and virulence of a mixed-species oral biofilm. PLoS Pathog. 8:e1002623. doi:10.1371/journal.ppat.1002623.

Yamashita Y, Bowen WH, Burne RA, Karamitsu HK. 1993. Role of the Streptococcus mutans gtf genes in caries induction in the specific-pathogen-free rat model. Infect Immun. $61: 3811-3817$. 